\title{
3 Quanten-Identität und Ununterscheidbarkeit
}

\author{
Holger Lyre
}

\section{Übersicht}

$3.1 \quad$ Quantentheorie gleichartiger Objekte . . . . . . . . . . . . . . . . . . . 79

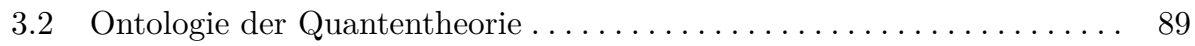

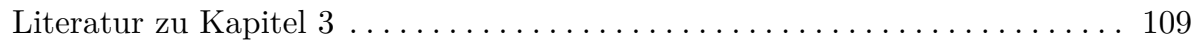

Kapitel 3 steht technisch und sachlich zwischen Kapitel 1 und 6. In Kapitel 1 wurde die 1-Teilchen-Quantenmechanik im Hilbertraum $\mathcal{H}$ eingeführt, das vorliegende Kapitel behandelt $n$ Teilchen im Tensorprodukt-Raum $\mathcal{H}_{n}$ und Kapitel 6 variable Teilchenzahlen mit Aufsteige- und Absteigeoperatoren im Fockraum $\mathcal{H}_{F}=\oplus \mathcal{H}_{n}$. Das Kapitel zerfällt in zwei Teile, wobei 3.1 physikalisch, 3.2 aber stärker philosophisch orientiert ist.

\subsection{Quantentheorie gleichartiger Objekte}

\subsubsection{Statistische Mechanik}

Die Quantentheorie hat ihren historischen Ursprung zu einem wesentlichen Teil in der Thermodynamik. Das Problem der Schwarzkörperstrahlung veranlasste Max Planck 1900 dazu, eine neue Ad-hoc-Regel über die Energieverteilung des Strahlungsfeldes eines schwarzen Körpers in Abhängigkeit von der Frequenz zu formulieren und dabei das Plancksche Wirkungsquantum als neue Naturkonstante einzuführen. Dieser Schritt wird allgemein als Geburtsstunde der Quantentheorie angesehen, und man kann sagen, dass das Problem der Schwarzkörperstrahlung eine Anomalie für die klassische Physik darstellt. Eine weitere Anomalie findet

sich im Fall der Mischungsentropie gleicher Gase - und dies führt auf das Thema des Kapitels: Sind physikalische Objekte Individuen, d.h., besitzen sie eine sowohl 
zeitüberbrückende, diachrone Identität als auch eine synchrone Identität im Sinne ihrer Unterscheidbarkeit von anderen Objekten?

Man betrachte hierzu zwei mit Gas gefüllte Kammern, die durch eine Trennwand separiert sind. Druck und Temperatur seien auf beiden Seiten gleich. Nach Herausnehmen der Trennwand mischen sich die Gase, die innere Energie des Gesamtsystems bleibt unverändert. Handelt es sich um verschiedene Gase, so ist der Prozess irreversibel, und die Entropie, eine Maßgröße für die Anzahl der Mikrozustände in einem gegebenen Makrozustand, steigt. Eine Mischung gleicher Gase sollte jedoch zu keinerlei Entropieanstieg führen, denn die Trennwand kann reversibel wieder eingefügt werden. Dieses Resultat steht in Einklang mit der makroskopischen Sichtweise der phänomenologischen Thermodynamik. Vom mikroskopischen Standpunkt der kinetischen Gastheorie bzw. statistischen Thermodynamik besteht das Gas jedoch aus Molekülen, die Entropie leitet sich nun aus der Maxwell-Bolzmann-Statistik ab und hängt von der Anzahl der Mikrozustände in einem Makrozustand ab. Werden zwei Gase gemischt, so ist es mikroskopisch scheinbar irrelevant, ob es sich um Moleküle einer oder verschiedener Gassorten handelt, denn in jedem Fall mischen sich die Moleküle ja individuell. Nach dieser Betrachtungsweise erhält man eine Erhöhung der Mischungsentropie auch für den Fall gleichartiger Gase auf beiden Seiten des Behälters - im Widerspruch zum Ergebnis der phänomenologischen Thermodynamik. Hierauf hatte Josiah Willard Gibbs Ende des 19. Jahrhunderts erstmals hingewiesen, das Problem ist als GibbsParadox bekannt.

Formal lässt sich der Widerspruch zum Verschwinden bringen, wenn bei der Zählung der Mikrozustände der Gibbssche Korrekturfaktor $\frac{1}{N !}$ verwendet wird (wobei $N$ die Teilchenzahl bezeichnet). Offensichtlich besagt dieser Faktor, dass die Zahl der Mikrozustände durch die Zahl ihrer Permutationen geteilt werden muss, was bedeutet, dass aus der Zählung der Mikrozustände die Möglichkeit der individuellen Erfassung eines Zustandes herausgerechnet wird.

Dies lässt sich anschaulich illustrieren. Betrachten wir die kombinatorisch möglichen Verteilungen zweier Teilchen $a$ und $b$ auf zwei Zustände, etwa Zustände verschiedener Energien, hier als Boxen dargestellt:

$$
\begin{array}{|c|c|}
\hline a b & \\
\hline & a b \\
\hline a & b \\
\hline b & a \\
\hline
\end{array}
$$

Im ersten und zweiten Fall befinden sich beide Teilchen jeweils im gleichen Energiezustand, im dritten und vierten Fall in unterschiedlichen Energien. Die Zählung der Besetzungszahlmöglichkeiten hängt nun davon ab, wie man die Fälle (3) und (4) gewichtet. Falls sich nämlich, wie durch den Gibbsschen Korrekturfaktor indiziert, die Objekte $a$ und $b$ nur bis auf Permutation bestimmen lassen, lassen 
sich die Fälle (3) und (4) nicht unterscheiden. Mit $\frac{1}{2 !}$ gewichtet wird hieraus in der Zählung nur ein Zustand. Insgesamt ergeben sich daher gegenüber den vier Möglichkeiten in (3.1) nur noch drei mögliche Besetzungen:

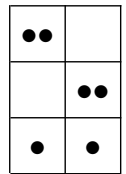

Die Notation $\bullet$ deutet an, dass die durch $a$ und $b$ vormals suggerierte eindeutige Erfassung der beiden Objekte aufgegeben wurde. De facto bedeutet dies, dass die beiden Objekte empirisch ununterscheidbar sind (jedenfalls hinsichtlich der Zählung physikalischer Besetzungsmöglichkeiten).

Die Verwendung des Gibbsschen Korrekturfaktors erwies sich in der Folge in allen Bereichen der statistischen Mechanik als unvermeidlich, um mit dem Experiment in Einklang stehende Resultate zu erzielen. Insbesondere erwies es sich als unerlässlich, bei der Konstruktion einer Quantenmechanik mehrerer Teilchen in den 20er Jahren des 20. Jahrhunderts ein Ununterscheidbarkeits-Postulat der folgenden Form zugrundezulegen:

Die Anwendung einer Teilchenpermutationen auf einen Viel-TeilchenZustand führt formal auf einen Zustand, der vom ursprünglichen Zustand physikalisch ununterscheidbar ist.

Die mathematische Umsetzung dieses Postulats liegt in der Forderung der Permutationssymmetrie quantenmechanischer Zustände, die wir in den beiden kommenden Abschnitten betrachten.

\subsubsection{Mehr-Teilchen-Tensorprodukt}

In Kapitel 1 wurde die Quantenmechanik eines Teilchens, oder allgemeiner: eines Objekts, betrachtet, nun fragen wir nach der Verallgemeinerung für Systeme aus beliebig vielen Objekten. Dabei werden wir uns auf gleichartige Teilchen kaprizieren, also solche, die von derselben Sorte sind wie beispielsweise mehrere Elektronen oder Photonen oder dergleichen. Allgemein besitzt ein quantenmechanisches Objekt mit $n$ Eigenzuständen Zustände, die als Vektoren eines $n$-dimensionalen Hilbertraums darstellbar sind. Setzt man ein $n$ - und ein $m$-dimensionales Objekt zu einem größeren Objekt, einem Compound-System, zusammen, so besitzt das Gesamtobjekt im Allgemeinen Zustände, die in einem $n \cdot m$-dimensionalen Hilbertraum definiert sind. Die Besonderheiten dieser Zusammensetzung gilt es zu verstehen.

Betrachten wir zunächst die Zusammensetzung von Objekten in der klassischen Physik. Hier ist der Zustandsraum eines Objekts durch den 6-dimensionalen Phasenraum gegeben. Er ist isomorph $\mathrm{zu} \mathbb{R}^{6}$ und wird von den je drei Orts- und 
Impulskoordinaten aufgespannt. Der Phasenraum eines Systems bestehend aus zwei Objekten ist entsprechend 12-dimensional und durch das direkte Produkt $\mathbb{R}^{6} \times \mathbb{R}^{6}=\mathbb{R}^{12}$ gegeben. Das direkte Produkt entspricht mengentheoretisch dem kartesischen Produkt, also der Menge aller geordneten Paare: $A \times B=\{(a, b) \mid a \in$ $A \wedge b \in B\}$. Für endlich viele Vektorräume stimmt das direkte Produkt mit der direkten Summe der Vektorräume überein: $\mathbb{R}^{n} \oplus \mathbb{R}^{m}=\mathbb{R}^{n+m}$. Hieraus ist ersichtlich, dass die Vektoren aus den einzelnen Teilräumen des Produktraums einfach „nebeneinandergestellt" werden, ohne dass irgendwelche Beziehungen zwischen diesen Vektoren bestünden. Physikalisch bedeutet dies, dass die Teilsysteme eines zusammengesetzten Systems in der klassischen Physik unabhängig sind und in keiner Weise korrelieren. Genau dies ändert sich bei der quantenmechanischen Zusammensetzung.

In der Quantenmechanik wird die Zusammensetzung von Systemen durch das Tensorprodukt ihrer Hilberträume beschrieben. Seien $\left\{\vec{e}_{i}\right\}$ und $\left\{\vec{h}_{j}\right\}$ Basissysteme zweier $n$ - und $m$-dimensionaler Hilberträume $\mathcal{H}^{n}$ und $\mathcal{H}^{m}$, dann bezeichnet der Ausdruck $\mathcal{H}^{n} \otimes \mathcal{H}^{m}$ das Tensorprodukt beider Vektorräume. Der Produktraum $\mathcal{H}^{n \cdot m}$ besitzt, anders als in der klassischen Physik, die Dimension $n \cdot m$ und wird von den Basisvektoren $\vec{e}_{i} \otimes \vec{h}_{j}$ aufgespannt. Der springende Punkt ist, dass sich ein allgemeiner Vektor $\vec{\psi}=\sum_{i, j} \alpha_{i j} \vec{e}_{i} \otimes \vec{h}_{j}$ eines Tensorproduktraums nicht als Produkt der Basisvektoren $\vec{e}_{i}$ und $\vec{h}_{j}$, sondern nur als lineare Superposition schreiben lässt. Bei der Zusammensetzung von Teilsystemen zu Gesamtsystemen via Tensorprodukt bestehen daher Beziehungen zwischen den Zuständen der Teilsysteme, so genannte Korrelationen, die durch die Superposition der Produktzustände der Teile im Gesamtsystem zustande kommen. Diese besondere Eigentümlichkeit der Quantenmechanik heißt Verschränkung und führt zu zahlreichen Besonderheiten, denen sich insbesondere Kapitel 4 widmen wird. Der Fokus des jetzigen Kapitels liegt auf der Konstruktion von Viel-Teilchen-Zuständen.

Aus dem vorher Gesagten ergibt sich unmittelbar, dass sich ein $n$-dimensionaler Hilbertraum als Tensorprodukt eindimensionaler Hilberträume schreiben lässt. Der Zustandsraum von $n$ Teilchen in der Quantenmechanik ist also das Tensorprodukt von $n$ Einteilchen-Hilberträumen $\mathcal{H}$ :

$$
\mathcal{H}^{n}=\mathcal{H}_{1} \otimes \mathcal{H}_{2} \otimes \ldots \otimes \mathcal{H}_{n}=\bigotimes_{i=1}^{n} \mathcal{H}_{i}
$$

Die einfachsten Basiszustände $\psi \in \mathcal{H}^{n}$ eines $n$-Teilchen-Hilbertraums lassen sich schreiben als

$$
\psi=\psi(1,2,3 \ldots n)=\psi_{1} \otimes \psi_{2} \otimes \ldots \otimes \psi_{n}, \quad \psi_{i} \in \mathcal{H}_{i}
$$

Aufgrund des in Abschnitt 3.1.1 eingeführten und heuristisch begründeten Ununterscheidbarkeits-Postulats gilt nun, dass jede Permutation gleichartiger 
Teilchen in einem Vielteilchenzustand zu einem physikalisch ununterscheidbaren Zustand führt. Bezeichnet $\hat{P}_{i j}$ eine Vertauschung der Teilchen $i$ und $j$, so ist der Zustand

$$
\hat{P}_{i j} \psi(1,2, \ldots i, \ldots j, \ldots n)=\psi(1,2, \ldots j, \ldots i, \ldots n)
$$

vom Zustand $\psi(1,2, \ldots i, \ldots j, \ldots n)$ physikalisch nicht zu unterscheiden ${ }^{1}$ Wegen der Nicht-Kommutativität des Tensorprodukts muss der Zustand eines Vielteilchensystems die allgemeine Form

$$
\Psi(1,2, . . n)=\sum_{P} \frac{C(n)}{\sqrt{n !}} \psi(1,2, \ldots n)
$$

haben, wobei auf der rechten Seite die Summe über $n$ ! Permutationen zu bilden ist (mit einem hier nicht näher zu bestimmenden und ggf. komplexen Faktor $C(n)$, der von der Teilchensorte abhängt).

Die Menge aller Permutationen einer geordneten Menge aus $n$ Elementen mit der Dimension $n$ ! wird Symmetrische Gruppe $S_{n}$ oder auch Permutationsgruppe genannt. Die Invarianz eines Zustands unter der Permutationsgruppe besagt, dass jede physikalische Observable $\hat{O}$ mit jedem Permutationsoperator $\hat{P}$ kommutiert; keine physikalische Messobservable kann daher zwischen einem permutierten und einem unpermutierten Zustand unterscheiden. Formal bedeutet dies

$$
\langle\psi|\hat{O}| \psi\rangle=\langle\hat{P} \psi|\hat{O}| \hat{P} \psi\rangle=\left\langle\psi\left|\hat{P}^{-1} \hat{O} \hat{P}\right| \psi\right\rangle \quad \text { bzw. } \quad[\hat{O}, \hat{P}]=0 .
$$

\subsubsection{Quantenstatistik}

Wie in 3.1.1 angedeutet, muss die Maxwell-Boltzmann-Statistik der klassischen statistischen Mechanik im Übergang zur Quantenstatistik durch eine neue Statistik ersetzt werden, die das Ununterscheidbarkeits-Postulat berücksichtigt. Es zeigt sich, dass die Natur diese Forderung auf dem Level der Elementarteilchen auf zweierlei Weisen realisiert - und zwar abhängig davon, ob die Teilchen halboder ganzzahligen Spin besitzen.

Dem indischen Physiker Satyendranath Bose gelang es 1924, Plancks Formel für die Energiedichte der Schwarzkörperstrahlung ohne Rückgriff auf die klassische Elektrodynamik, wie vormals Planck, sondern allein gestützt auf die Annahme von Lichquanten mit Phasenraumvolumina $h^{3}$ abzuleiten (vgl. Darrigol, 1991). In die Besetzungszahlen der Zustände ging dabei der Gibbsche Korrekturfaktor ein. Bose bat Einstein, der die Lichtquantenhypothese 1905 aufgebracht hatte, um Hilfestellung bei der Publikation. Einstein erkannte die Bedeutung der Arbeiten

\footnotetext{
${ }^{1}$ Der Permutationsoperator $\hat{P}_{i j}$ ist selbstadjungiert und hat die spezielle Eigenheit, dass seine Eigenwerte 1 und -1 sind (vgl., analog, Abschnitt 1.2.4).
} 
Boses und fügte eigene Ergänzungen hinzu. Nach Schrödingers Wellenmechanik von 1926 wurde die Allgemeinheit der Bose-Einstein-Statistik für VielteilchenWellenfunktionen erkannt, die Teilchen mit ganzzahligem Spin beschreiben. Formal liegt der Bose-Einstein-Statistik das Ununterscheidbarkeits-Postulat zugrunde, was auf die Permutationsinvarianz bosonischer Zustände unter der Transformation 3.4 führt.

Da nicht $\psi$, sondern $|\psi|^{2}$ mit observablen Größen verknüpft ist, liegt die Wellenfunktion nur bis auf einen Phasenfaktor fest. Ferner gilt für den Permutationsoperator $\hat{P}_{i j}^{2}=1$, er besitzt also die Eigenwerte \pm 1 (siehe Fußnote 1). Neben 3.4 lässt dies daher auch die Möglichkeit zu, dass eine Permutation im Gegensatz zu (3.4) auf einen Vorzeichenwechsel führt und antisymmetrisch ist:

$$
\hat{P}_{i j} \psi(1,2, \ldots i, \ldots j, \ldots n)=-\psi(1,2, \ldots j, \ldots i, \ldots n) .
$$

Wiederum zeigt sich empirisch, dass antisymmetrische Wellenfunktionen mit einer bestimmten Sorte von Teilchen verknüpft sind, nämlich solchen, die halbzahligen Spin besitzen. Wolfgang Pauli hatte 1925 zur quantentheoretischen Erklärung des Aufbaus der Atome bereits die Forderung aufgestellt, dass keine zwei Elektronen in einem Atom in allen vier Quantenzahlen, die der Zustandsbeschreibung eines Elektrons im Orbital dienen, übereinstimmen dürfen. Dieses so genannte Paulische Ausschließungsprinzip führt im Zusammenspiel mit dem UnunterscheidbarkeitsPostulat zu der Forderung, dass die Mehrelektronen-Wellenfunktion gemäß 3.6 antisymmetrisch unter der Vertauschung zweier Elektronen ist.

Die Verallgemeinerung dieser Forderung für beliebige Teilchen mit halbzahligem Spin, also Fermionen, führt zur Fermi-Dirac-Statistik. Anschaulich besagt die Fermi-Dirac-Statistik, dass etwa im Falle eines Systems zweier gleichartiger Teilchen nur die dritte Zustandsmöglichkeit in 3.2 erlaubt ist, wobei die Wellenfunktion antisymmetrisch zu schreiben ist:

$$
\Psi_{a}=\frac{1}{\sqrt{2}}\left(\psi_{a} \psi_{b}-\psi_{b} \psi_{a}\right)
$$

Demgegenüber gestattet Bose-Einstein alle drei Möglichkeiten in Form symmetrisierter Wellenfunktionen

$$
\begin{aligned}
\Psi_{s}^{(1)} & =\psi_{a} \psi_{a} \\
\Psi_{s}^{(2)} & =\frac{1}{\sqrt{2}}\left(\psi_{a} \psi_{b}+\psi_{b} \psi_{a}\right), \\
\Psi_{s}^{(3)} & =\psi_{b} \psi_{b} .
\end{aligned}
$$

Bose-Einstein- und Fermi-Dirac-Statistik stellen die beiden gesuchten neuen Quantenstatistiken zur Berechnung der Besetzungszahlen quantenmechanischer Systeme dar, die die klassische Maxwell-Boltzmann-Statistik ablösen. Hierzu zwei ergänzende Anmerkungen. Erstens, die Forderung, dass die Vertauschung zweier Teilchen in einer Mehrteilchen-Wellenfunktion entweder symmetrisch 3.4 
oder antisymmetrisch (3.6) zu erfolgen hat, besagt nicht, dass sämtliche möglichen Zwei-Teilchen-Transpositionen in einem Mehrteilchen-Zustand entweder nur symmetrisch oder nur antisymmetrisch zu erfolgen haben. Rein formal lassen sich auch gemischt-symmetrische Permutationen betrachten, also solche, die einige Teilchen symmetrisch, andere antisymmetrisch transformieren. Dies führt auf so genannte Para-Statistiken (vgl. Messiah und Greenberg, 1964). Bislang zeigt sich jedoch empirisch, dass solcherart gemischt-symmetrische Darstellungen der Permutationsgruppe in der Natur nicht realisiert sind. Die Möglichkeit para-statistischer Zustände soll hier daher nicht weiter verfolgt werden, eine Mehrteilchen-Wellenfunktion ist entweder total symmetrisiert oder total antisymmetrisiert zu schreiben.

Zweitens zeigt sich - ebenfalls rein empirisch -, dass nach (3.4) symmetrisierte Zustandsfunktionen Teilchen mit ganzzahligem Spin, also Bosonen, und nach (3.6) antisymmetrisierte Funktionen Teilchen mit halbzahligem Spin, also Fermionen, beschreiben. Eine tieferliegende, theoretische Begründung für den Zusammenhang zwischen Spinzahl und Statistik wurde erst Ende der 30er Jahre durch Fierz und Pauli in Form des Spin-Statistik-Theorems gegeben (und seither in unterschiedlichen Formen von verschiedenen Autoren). Die Beweise beruhen typischerweise auf sehr allgemeinen Annahmen der relativistischen Quantenfeldtheorie wie Lokalität und positiver Definitheit der Energie, die den gegebenen Kontext übersteigen (siehe Kapitel 6).

Die empirische Relevanz der Fermi- und der Bose-Statistik zeigt sich handfest in Teilchen-Streuexperimenten, wie Feynman in seinen berühmten Lectures sehr anschaulich ausführt (Feynman et al. 1964 , Kap. 4). Wir betrachten zunächst zwei empirisch unterscheidbare Teilchen $a$ und $b$. Teilchen $a$ streut in Richtung 1 mit Amplitude $a_{1}=\langle a \mid 1\rangle$ und Teilchen $b$ in Richtung 2 mit Amplitude $b_{2}=\langle b \mid 2\rangle$. Die Wahrscheinlichkeit für die gemeinsame Doppelstreuung ist das Produkt der Einzelwahrscheinlichkeiten $\left|a_{1}\right|^{2}\left|b_{2}\right|^{2}$. Falls umgekehrt $a$ in Richtung 2 und $b$ in Richtung 1 streut, erhält man $\left|a_{2}\right|^{2}\left|b_{1}\right|^{2}$, und für die Gesamtwahrscheinlichkeit

$$
P=\left|a_{1}\right|^{2}\left|b_{2}\right|^{2}+\left|a_{2}\right|^{2}\left|b_{1}\right|^{2} \text {. }
$$

Nun sei angenommen, die Richtungen 1 und 2 näherten sich einander an, dann reduziert sich der obige Ausdruck mit $a=a_{1}=a_{2}$ und $b=b_{1}=b_{2}$ auf

$$
P=2|a|^{2}|b|^{2} \text {. }
$$

Falls $a$ und $b$ nun ununterscheidbare Bosonen sind, lässt sich zwischen dem Doppelstreuprozess $a$ in Richtung 1 und $b$ in Richtung 2 sowie dem Austauschprozess $a$ in Richtung 2 und $b$ in Richtung 1 empirisch nicht unterscheiden, und man erhält (gemäß der Regel „sum before you square“)

$$
P_{b}=\left|a_{1} b_{2}+a_{2} b_{1}\right|^{2}=4|a|^{2}|b|^{2},
$$


also eine doppelt so hohe Wahrscheinlichkeit wie im Falle unterscheidbarer Teilchen. Für Fermionen muss andererseits die totale Übergangsamplitude als antisymmetrisiert angesetzt werden,

$$
a_{1} b_{2}-a_{2} b_{1}
$$

so dass im Falle angeglichener Richtungen 1 und 2

$$
P_{f}=0
$$

folgt. Es ist unmöglich, zwei ununterscheidbare Fermionen im gleichen StreuEndzustand zu erhalten.

Eine weitere bemerkenswerte Konsequenz der Fermi-Dirac-Statistik ist, dass man aus dem Ausschließungsprinzip für ein Fermionen-Gas bei hoher Dichte den sogenannten Fermi- oder Entartungsdruck ableiten kann. In einem Weißen Zwerg wirkt dieser Fermi-Druck einer weiteren Kompression durch die Gravitation entgegen und stabilisiert auf diese Weise den Stern. Mindestens zwei interessante physik-philosophische Fragen stellen sich in diesem Zusammenhang, die hier aber nur genannt und nicht beantwortet werden können. Nämlich erstens: In welcher Weise ist der Fermi-Druck wirklich ein Druck? Schließlich hat er seine Grundlage in einer Symmetrie-Forderung, nicht in der Existenz einer expliziten Wechselwirkung (wie beispielsweise der Gravitationsdruck). Und zweitens: Inwieweit könnte dies bereits auf einen ontologischen Unterschied von Fermionen und Bosonen hindeuten?

\subsubsection{Symmetrische Gruppe}

Das Ununterscheidbarkeits-Postulat wirkt als Superauswahlregel auf dem Raum (3.3) der Zustände gleichartiger Objekte. Es besagt, dass $\mathcal{H}^{n}$ in Teilräume oder Sektoren zerfällt, die den Darstellungen der Permutationsgruppe entsprechen. Im Zusammenspiel mit den beiden vorgängigen, empirischen Bemerkungen führt das Ununterscheidbarkeits-Postulat auf folgendes Symmetrisierungspostulat (vgl. Messiah, 1979, Kap. 14):

Der Zustandsraum (3.3) der Quantentheorie n gleichartiger Objekte zerfällt in den Unterraum aller vollständig symmetrischen, bosonischen und den aller vollständig anti-symmetrischen, fermionischen Zustandsfunktionen.

Kompakte Erläuterungen zu Gruppendarstellungen und Auswahlregeln finden sich in der grauen Box auf Seite 87, hier sei ein einfaches Beispiel betrachtet: Da ein Quantenobjekt mit nur zwei Zuständen einer irreduziblen Darstellung der $S U(2)$ entspricht, spannen die Zustände (3.7) und 3.8 - 3.10 die Unterräume des Tensorprodukts zweier irreduzibler $S U(2)$-Darstellungen auf. Man sieht daran, dass 


\section{Gruppendarstellungen und Auswahlregeln}

Unter der Vektorraum-Darstellung einer Gruppe $G$ versteht man eine homomorphe Abbildung von $G$ in eine Automorphismengruppe nicht-singulärer Operatoren auf einem Vektorraum $\mathbb{V}$. Unter der Gruppenwirkung zerfällt $\mathbb{V}$ in invariante Unterräume (Sektoren), was anschaulich bedeutet, dass die Anwendung der Gruppenoperatoren auf Zustände, die den Darstellungsraum aufspannen, nicht aus diesem Raum herausführt, dass also die Zustände nur unter sich selbst transformieren. Es sind daher keine Zustandsübergänge von einem Sektor in einen anderen möglich. Die Darstellung bzw. der Darstellungsraum heißt irreduzibel, falls keine weiteren, unter der Gruppenwirkung invarianten Unterräume existieren (mit Ausnahme des Nullvektors und $\mathbb{V}$ selbst als trivialen Unterräumen).

Ist der Darstellungsraum entartet, spricht man von einem Multiplett. Ein Zustand heißt entartet, falls zu einem Eigenwert des Hamilton-Operators mehrere Eigenfunktionen gehören. Ein Multiplett ist also ein Satz von entarteten Zuständen mit dem gleichen Energieeigenwert, der Entartungsgrad entspricht der Dimension der Darstellung. Der Hamilton-Operator ist dabei invariant unter der Gruppe, kommutiert also mit allen Gruppenoperatoren. Für die Permutationsgruppe haben wir dies schon am Ende von 3.1.2 gesehen.

Auswahlregeln geben Auskunft darüber, ob bestimmte Übergänge zwischen den Zuständen erlaubt sind oder nicht. Verbotene Übergänge entsprechen verschwindenden Übergangswahrscheinlichkeiten für die entsprechenden Störoperatoren $\hat{A}$ :

$$
\langle\psi|\hat{A}| \phi\rangle=\langle\phi|\hat{A}| \psi\rangle=0 .
$$

Eine Einschränkung der Zustandsübergänge kann dadurch entstehen, dass der Zustandsraum gewissen Symmetrien genügt und damit Erhaltungsgrößen kennzeichnet. So induzieren Drehimpuls- und Spinerhaltung der Atome die verschiedenen Auswahlregeln zur Berechnung der Spektrallinien in der Atomphysik. Auswahlregeln charakterisieren somit auch die Zerlegung des Hilbertraums in inkohärente Sektoren, deren Zustände nicht superponierbar sind. Falls die Regeln sich nicht nur auf bestimmte Operatoren, sondern auf alle Messobservablen $\hat{A}$ beziehen, spricht man von Superauswahlregeln. Superauswahlregeln kennzeichnen streng erhaltene Quantenzahlen - im Falle der Permutationssymmetrie die Bosonen- und die Fermionenzahl. Im Rahmen der Supersymmetrie werden diese Quantenzahlen aufgegeben und stattdessen Übergänge zwischen Bosonen und Fermionen postuliert. Hierbei handelt es sich bislang jedoch nur um eine hypothetische Symmetrie. 
der Tensorproduktraum in einen eindimensionalen und einen dreidimensionalen, irreduziblen Unterraum zerfällt. Oder anders gesagt: Das Tensorprodukt zweier fundamentaler $S U(2)$-Darstellungen wird zerlegt in die direkte Summe eines Singuletts und eines Tripletts, schematisch notierbar als

$$
(2) \otimes(2)=(1) \oplus(3)
$$

Nun interessieren wir uns hier für die Darstellungen der $S_{n}$, diese hängen jedoch mit der $S U(n)$ zusammen. Während (3.7) eine eindimensionale (antisymmetrische) Darstellung der $S_{2}$ bildet, spannen (3.8) -3.10 einen dreidimensionalen (symmetrischen) Darstellungsraum auf. Letzterer ist reduzibel, denn jeder der Zustände (3.8)-3.10 entspricht einer irreduziblen eindimensionalen Darstellung. Allgemein gilt, dass sämtliche total symmetrischen und total antisymmetrischen irreduziblen Darstellungen der $S_{n}$ eindimensional sind (in unserem Beispiel führt die Anwendung des Permutationsoperators auf jeden der Zustände (3.7)- 3.10 nicht aus dem eindimensionalen Strahl $c \psi$, wobei $\psi$ einer der Zustände (3.7)-(3.10) ist, heraus). Demgegenüber sind die irreduziblen gemischt-symmetrischen Darstellungen, die ab $S_{3}$ auftreten, höherdimensional. So zerlegt die $S_{3}$ den Zustandsraum $H^{3}$ dreier Teilchen irreduzibel in je eine total symmetrische und eine total antisymmetrische sowie zwei zweidimensionale gemischt-symmetrische Darstellungen, also Dubletts (die wir hier nicht betrachten) ${ }^{2}$

Was sich hier am Beispiel des Zusammenhangs von $S_{2}$ und $S U(2)$ andeutet, gilt allgemein: Die Multiplizität einer irreduziblen Darstellung der $S_{n}$ ist gleich der Dimension der irreduziblen Darstellung der $S U(n)$ und umgekehrt ${ }^{3}$ Die Darstellungen von $S_{n}$ und $S U(n)$ lassen sich graphisch elegant durch die so genannten Young-Schemata (auch Young-Tableaux) illustrieren, was hier leider nicht ausge-

${ }^{2}$ Die Zustandsfunktionen lauten explizit

$$
\Psi_{S}=\frac{1}{\sqrt{6}}\left(\psi_{a} \psi_{b} \psi_{c}+\psi_{b} \psi_{a} \psi_{c}+\psi_{c} \psi_{b} \psi_{a}+\psi_{a} \psi_{c} \psi_{b}+\psi_{c} \psi_{a} \psi_{b}+\psi_{b} \psi_{c} \psi_{a}\right)
$$

für das symmetrische und

$$
\Psi_{A}=\frac{1}{\sqrt{6}}\left(\psi_{a} \psi_{b} \psi_{c}-\psi_{b} \psi_{a} \psi_{c}-\psi_{c} \psi_{b} \psi_{a}-\psi_{a} \psi_{c} \psi_{b}+\psi_{c} \psi_{a} \psi_{b}+\psi_{b} \psi_{c} \psi_{a}\right)
$$

für das antisymmetrische Singulett. Man überzeugt sich leicht, dass die Vertauschung zweier Objekte in $\Psi_{A}$ zu einem Vorzeichenwechsel führt.

${ }^{3}$ Einige zusätzliche Beispiele ohne weiteren Kommentar: Das Tensorprodukt dreier fundamentaler $S U(2)$-Dubletts zerfällt in ein Dublett und ein Quartett: $(2) \otimes(2) \otimes(2)=(2) \oplus(4)$. Das Tensorprodukt zweier fundamentaler $S U(3)$-Tripletts zerfällt in ein Triplett und ein Sextett: $(3) \otimes(3)=(3) \oplus(6)$. Und für das Tensorprodukt dreier fundamentaler $S U(3)$-Tripletts erhält man: $(3) \otimes(3) \otimes(3)=(1) \oplus(8) \oplus(8) \oplus(10)$. Entsprechend besitzt die $S_{3} 1$ eindimensionale antisymmetrische, 8 zweidimensionale gemischt-symmetrische und 10 eindimensionale symmetrische irreduzible Darstellungen. 
führt werden kann (siehe hierzu Messiah, 1979, Anhang D.4, sowie bereits Weyl, 1928, Kap. V, § 13).

\subsection{Ontologie der Quantentheorie}

In 3.1 wurde auf die Konsequenzen des empirischen Faktums der physikalischen Ununterscheidbarkeit von Teilchen oder Objekten im formalen Apparat der Quantentheorie eingegangen. Nun sollen die Implikationen dieses Faktums in Hinblick auf Ontologie (oder auch zeitgenössische Metaphysik) diskutiert werden. Als Ontologie bezeichnet man diejenige Teildisziplin der Philosophie, die nach dem Sein und den Seinsformen fragt, also danach, was existiert, und den Arten und Weisen, wie es existiert (siehe Loux (1998) als empfehlenswerte Einführung in die moderne Ontologie, Castellani (1998) als nützliche Textsammlung und insbesondere French und Krause (2006) als umfassende Darstellung der nachfolgend behandelten Fragen der Quanten-Ontologie).

\subsubsection{Identität und Leibniz-Prinzip}

\section{Identität und Individualität}

Im Zentrum der Ontologie stehen Fragen nach Identität und Individualität 4 nicht wenige Philosophen koppeln die Möglichkeit einer Ontologie sogar vollständig an die Frage der Identität bzw. daran, wie sich Entitäten individuieren lassen. Notorisch ist in diesem Zusammenhang Willard van Orman Quines bekanntes Diktum „No entity without identity" (Quine, 1969, S. 23).

Vorab ein paar Bemerkungen zur Terminologie: Als Entität bezeichnet man in der Philosophie jedwede Seinsform, ob konkret oder abstrakt 5 Physikern geht es häufig darum, physikalische Systeme zu beschreiben. Der Ausdruck System wird dabei meist so verwendet, dass ein System auch aus Teilsystemen bestehen kann. Elementare, nicht weiter zerlegbare Systeme sind zum Beispiel Elementarteilchen. Will man sich nicht von vornherein auf eine Teilchenontologie festlegen, lässt sich

\footnotetext{
${ }^{4}$ Beide Begriffe werden hier weitestgehend synonym gebraucht (Vorsicht ist allerdings geboten bei den Zitaten in Abschnitt 3.2.2.

${ }^{5}$ Als Konkreta oder Partikularia bezeichnet man Entitäten mit raumzeitlicher Lokalisierung, worunter insbesondere sämtliche Alltagsgegenstände und sonstige physikalische Dinge fallen. Abstrakta existieren demgegenüber nicht in Raum und Zeit und sind typischerweise kausal inert. Zu den Standardbeispielen gehören Mengen, Zahlen, Propositionen, mögliche Welten oder abstrakte Konzepte wie Liebe, Gott oder das Gute. Aber auch Eigenschaften, soweit sie als Universalia konzipiert sind, sind abstrakt. Ihnen kommt aber die Möglichkeit zu, an Raumzeit-Stellen instantiiert zu sein.
} 
allgemeiner von physikalischen Objekten sprechen. Die Frage dieses Kapitels lautet in voller Allgemeinheit, was die numerische Distinktheit von Quantenobjekten ausmacht und wie sie sich individuieren lassen.

$\mathrm{Zu}$ beachten ist auch der Gebrauch des Begriffs Identität. In Physikbüchern ist gelegentlich von identischen Teilchen anstelle von ununterscheidbaren Teilchen die Rede. Eine solche Redeweise ist verwirrend, denn wenn mehrere Teilchen identisch sind, sind es ja eben nicht mehrere, sondern ein Teilchen. David Lewis bringt dies in unnachahmlicher Weise auf den Punkt:

Identity is utterly simple and unproblematic. Everything is identical to itself; nothing is ever identical to anything else except itself. There is never any problem about what makes something identical to itself; nothing can ever fail to be. And there is never any problem about what makes two things identical; two things never can be identical. (Lewis, 1986, 192-193)

Uns geht es in diesem Sinne um die Frage empirisch ununterscheidbarer Objekte und der damit zusammenhängenden Konzeption der Objekt-Identität - die Rede von gleichartigen Objekten ist daher zutreffender, wenn auch leider weniger gebräuchlich.

Es bieten sich drei Arten der Individuation physikalischer Objekte an, nämlich durch

(1) Mengen von Eigenschaften,

(2) raumzeitliche Lokalisation bzw. Raumzeit-Trajektorien,

(3) primitive (irreduzible) metaphysische Identität.

Betrachten wir zwei Teilchen $a$ und $b$ mit Raumzeit-Trajektorien $\gamma_{a}$ und $\gamma_{b}$ in einem gegebenen Raumvolumen. Falls die Teilchen von unterschiedlichen Sorten, also ungleichartig sind, gibt es wenigstens eine Eigenschaft, hinsichtlich derer sie sich unterscheiden ${ }^{6}$ Um Schwierigkeiten mit zusammengesetzten und aus elementareren Eigenschaften abgeleiteten Eigenschaften zu vermeiden, können wir uns

\footnotetext{
${ }^{6}$ Eigenschaften seien hier zunächst als im Prinzip empirisch erfassbar verstanden (wenn auch nicht unbedingt direkt beobachtbar). In einer empirischen Wissenschaft wie der Physik scheint es vordergründig immer um derartige Eigenschaften zu gehen, in der Metaphysik lassen sich Eigenschaften jedoch auch jenseits ihrer Empirizität noch grundlegender unterscheiden, wie etwa die Unterscheidung zwischen Eigenschaften als Universalien und als Tropen andeutet - hierauf wird weiter unten eingegangen. Zunächst seien Eigenschaften im Sinne der Physik aber als an Raumzeitpunkten instantiierte Universalia, also in re verstanden. Zur Erläuterung: Da Universalia, wie in Fußnote 5 hervorgehoben, Abstrakta sind, lassen sich im Prinzip auch nicht-instantiierte Eigenschaften, so genannte Universalia ante rem, betrachten. Die Eigenschaft, ein Einhorn zu sein, wäre ein Beispiel dafür. Strenge Universalienrealisten nehmen derartige Eigenschaften (aus Gründen, die wir hier nicht diskutieren können), in ihre Ontologie auf. Von diesen Möglichkeiten sei hier abgesehen.
} 
hier auf die fundamentalen Eigenschaften von Elementarteilchen, also Ruhemasse, Ladung und Spin kaprizieren. Die Unterscheidung eines, sagen wir, Elektrons von einem Photon bereitet aufgrund verschiedener Fundamentaleigenschaften keine Schwierigkeiten, dies wäre ein Beispiel für Individuation im Sinne von (1).

Was aber, wenn $a$ und $b$ gleichartig, also zum Beispiel zwei Elektronen, sind? In dem Fall könnten wir versuchen, die raumzeitliche Lokalisation zur synchronen Unterscheidung heranzuziehen. Die Individuation im Sinne von (2) besagt, dass die Raumzeit-Trajektorie der diachronen Identitätsstiftung dient. Schopenhauer sprach im Anschluss an Kant vom Raum als principium individuationis. Doch schon Kant bemerkte, dass die raumzeitliche Individuation einer weiteren Annahme bedarf, nämlich der Undurchdringbarkeit der betrachteten Objekte 7

Angenommen, die Objekte $a$ und $b$ könnten einander vollständig durchdringen, so dass sie fortan dieselbe Raumzeitstelle oder, bei ausgedehnten Objekten, Raumzeitregion einnehmen (vgl. auch Della Rocca, 2005). Nennen wir die an dieser Stelle oder Region befindliche Entität $X$. Welche Berechtigung hätten wir dann, von $X$ zu sagen, es handele sich um zwei Objekte im Gegensatz zu einem - oder auch zu tausend - Objekten? Falls wir ausschließlich im Sinne von (2) individuieren, müssten wir strenggenommen sagen, $a$ und $b$ verlieren ab dem Moment der vollständigen Durchdringung ihre Identität, statt dessen entsteht das neue Objekt $X$. Wir könnten dem nur entgehen, falls wir zusätzlich zu (2) die Impenetrabilität der Objekte fordern. Dass ferner auch die Topologie der Raumzeit oder konventionalistische Elemente bezüglich der Begründung der physikalischen Topologie und Geometrie eine Rolle spielen, wird am Ende des nächsten Abschnitts ausgeführt.

7 In den Vorlesungen über die Metaphysik, herausgegeben von Pölitz 1821, schreibt Kant über den Raum als Individuationsprinzip: „Die Gegenstände im Raum sind darum schon plura, weil sie im Raume sind“ (PM 66). Die dann folgende Betrachtung zweier Wassertropfen findet sich auch im Anhang zur „Amphibolie der Reflexionsbegriffe“ der Kritik der reinen Vernunft (A 1781/B 1787):

... ist doch die Verschiedenheit der Örter [...] zu gleicher Zeit ein genugsamer Grund der numerischen Verschiedenheit des Gegenstandes (der Sinne) selbst. So kann man bei zwei Tropfen Wasser von aller innern Verschiedenheit (der Qualität und Quantität) völlig abstrahiren, und es ist genug, daß sie in verschiedenen Örtern zugleich angeschaut werden, um sie für numerisch verschieden zu halten. (A263/B319)

Kants Position lässt sich natürlich nur vor dem Hintergrund seiner Transzendentalphilosophie verstehen, auf die hier nicht eingegangen werden kann und nach der sich die Physik nicht auf die Dinge an sich bezieht, sondern nur darauf, wie die Dinge uns unter Maßgabe der Anschauungsformen von Raum und Zeit und der Verstandeskategorien erscheinen. Zur Impenetrabilität findet sich in den Metaphysischen Anfangsgründen der Naturwissenschaft von 1786 der Lehrsatz 3 im zweiten Teil zur Dynamik: „Die Materie kann ins Unendliche zusammengedrückt, aber niemals von einer Materie, wie groß auch die drückende Kraft derselben sei, durchdrungen werden." (AA IV:501) 


\section{Das Leibniz-Prinzip}

Man könnte einwenden, dass Orte und Abstände, also Lokalisation im Raum, zu den Eigenschaften der Objekte hinzuzählen. Individuation im Sinne von (2) reduziert sich dann auf Individuation im Sinne von (1). Wir wollen daher die Idee, Identität durch Eigenschaftsgleichheit festzulegen, näher verfolgen. Diese Idee liegt Gottfried Wilhelm Leibniz' bekanntem Prinzip der Identität des Ununterscheidbaren zugrunde (gängigerweise abgekürzt als PII: Principium identitatis indiscernibilium $)^{8}$

Für alle Eigenschaften F und Objekte x, y gilt: Wenn x die Eigenschaft

$\mathrm{F}$ dann und nur dann hat, wenn $\mathrm{y} F$ hat, dann ist $\mathrm{x}$ mit $\mathrm{y}$ identisch.

In formaler Notation lautet das PII:

$$
\forall F \forall x, y: \quad(F x \leftrightarrow F y) \Rightarrow(x=y)
$$

Leibniz hatte sein Prinzip so verstanden, dass es sich bei den Eigenschaften, über die quantifiziert wird, um monadische und intrinsische Eigenschaften handelt. Eine Eigenschaft ist intrinsisch, falls sie einem Objekt unabhängig von der Existenz anderer Objekte und Eigenschaften zukommt, andernfalls ist sie extrinsisch. Generische Kandidaten, wenn auch keineswegs unkontrovers, für intrinsische Eigenschaften sind Masse, Ladung und Spin bei Elementarteilchen. Extrinsische oder relationale Eigenschaften, kurz: Relationen, hängen von mehreren Entitäten ab und sind insofern mehrstellig. Die Relationen „größer als“ oder „Bruder von" sind paradigmatische Beispiele für zweistellige Relationen, „liegt zwischen“ ist dreistellig. Monadische Eigenschaften sind demgegenüber einstellig.

Im Lichte des obigen Lewis-Zitats besitzt unsere Formulierung des PII den Klang des Paradoxen, da zunächst von zwei Objekten x und y ausgegangen wird, die dann als identisch behauptet werden. Um dies zu vermeiden, sollte das PII eher in Form der logisch äquivalenten, kontrafaktischen Formulierung der Ungleichheit des Verschiedenen ausgedrückt werden:

\footnotetext{
${ }^{8}$ In den Primae veritates schreibt Leibniz: „Sequitur etiam hinc non dari posse duas res singulares solo numero differentes" (es folgt sogar, dass es keine zwei Einzeldinge geben kann, die sich lediglich numerisch unterscheiden). Die Fortführung des Zitats zeigt zugleich, dass Leibniz das PII als Folge eines von ihm als noch grundlegender angesehenen Prinzips angesehen hat, des Prinzips vom zureichenden Grunde: „utique enim oportet rationem reddi posse cur sint diversae, quae ex aliqua in ipsis differentia petenda est" (denn es muss möglich sein, einen Grund anzugeben, warum sie verschieden sind, was in irgendeinem Unterschiede in ihnen aufgesucht werden muss). Die Bedeutung des PII illustrierte Leibniz nach eigenem Bekunden ganz anschaulich den Damen am Schloss Herrenhausen, indem er sie aufforderte, zwei gleiche Blätter zu finden, was ihnen nicht gelang (vgl. C. I. Gerhardt (Hg.), Gottfried Wilhelm Leibniz: Philosophische Schriften. 7 Bände, Berlin 1875-1890. Nachdruck: Olms, Hildesheim, 1960, 214).
} 
Die Objekte $\mathrm{x}$ und $\mathrm{y}$ sind verschieden, falls $\mathrm{x}$ wenigstens eine Eigenschaft besitzt, die y nicht besitzt oder umgekehrt.

Oder einfacher: Keine zwei Objekte teilen alle Eigenschaften, formal:

$$
\forall F \nexists x, y: \quad(x \neq y) \wedge(F x \leftrightarrow F y)
$$

was mit 3.11 äquivalent ist 9

Die Frage stellt sich, inwieweit das PII eine unmittelbar evidente und gegebenenfalls a priori einsichtige metaphysische Forderung darstellt. Es ist hierzu instruktiv, sich die logische Umkehrung des PII, das Prinzip der Ununterscheidbarkeit des Identischen, vor Augen zu führen: $\forall F \forall x, y:(x=y) \Rightarrow(F x \leftrightarrow F y)$ bzw.

$$
\forall F \forall x, y: \quad(F x \nLeftarrow F y) \Rightarrow(x \neq y) .
$$

In Worten: Unterscheidbare Dinge können nie identisch sein. Diese Forderung scheint in der Tat unmittelbar evident. Man kann sich schwerlich mögliche Welten vorstellen oder konstruieren, die dieses Prinzip verletzen 10 Es handelt sich um einen guten Kandidaten für ein in allen metaphysisch möglischen Welten gültiges Prinzip.

Für das PII scheint dies jedoch nicht zu gelten, denn es ist ohne Weiteres möglich, Welten anzugeben, in denen das PII verletzt ist. Eine solche Welt, also ein Gegenbeispiel zu PII, hat Max Black anhand eines bekannten Szenarios diskutiert (das der Sache nach eine erweiterte Neuauflage von Kants Wassertropfen in Fußnote 7 darstellt). In Blacks eigenen Worten:

Isn't it logically possible that the universe should have contained nothing but two exactly similar spheres? We might suppose that each

${ }^{9}$ Dies zeigt man wie folgt:

$$
\begin{array}{lll} 
& \forall F \neg \exists x, y: \neg(x=y) \wedge(F x \leftrightarrow F y) \\
\Longleftrightarrow & \forall F \forall x, y: \quad \neg(\neg(x=y) \wedge(F x \leftrightarrow F y)) \\
\Longleftrightarrow & \forall F \forall x, y: \quad(x=y) \vee \neg(F x \leftrightarrow F y) \\
\Longleftrightarrow & \forall F \forall x, y: \quad(F x \leftrightarrow F y) \Rightarrow(x=y)
\end{array}
$$

\footnotetext{
${ }^{10}$ David Lewis vertritt in seinem modalen Realismus bezüglich möglicher Welten die Auffassung, dass es in anderen möglichen Welten Gegenstücke (counterparts) zu in der aktualen Welt befindlichen Entitäten gibt (etwa auch zu jedem Leser dieser Zeilen), nicht aber, wie beispielsweise Plantinga, dass Transwelt-Identität möglich ist, dass also eine Person in dieser Welt identisch ist mit Personen in anderen möglichen Welten (von denen wir reden, wenn wir Dinge sagen wie: ,ich wäre beinahe getroffen worden, konnte aber rechtzeitig zur Seite springen“). Counterparts hängen über die Beziehung der Ähnlichkeit miteinander zusammen, sie können einander beliebig ähnlich, nicht aber identisch sein. Vertreter der Transwelt-Identität verletzen demgegenüber, so Lewis, das Prinzip der Ununterscheidbarkeit des Identischen (vgl. Lewis, 1986, 198ff; Loux 1998 166ff).
} 
was made of chemically pure iron, had a diameter of one mile, that they had the same temperature, color, and so on, and that nothing else existed. Then every quality and relational characteristic of the one would also be a property of the other. (Black, 1952, 156)

Blacks Szenario geht von der Voraussetzung aus, dass die Raumzeitstelle nicht zur Individuation der beiden Kugeln herangezogen werden kann. Eine solche Voraussetzung ist beispielsweise in einer Welt erfüllt, in der der Raum relational ist. Ein Relationalismus des Raumes besagt, dass der Raum nichts als die Menge möglicher Körperrelationen ist, und dass daher ein leerer Raum unmöglich ist. Demgegenüber steht eine substantialistische Raum-Ontologie, die den Raum oder dessen Konstituenten als Entitäten sui generis ansieht. Während in einem relationalen Raum Orte und Abstände lediglich relationale Eigenschaften der in ihm befindlichen Objekte darstellen, besitzt ein Raumpunkt in einem substantialistischen Raum seinen Ort intrinsisch; im Prinzip existiert daher auch ein absolutes Bezugssystem im Raum 11

Wie am Ende des vorigen Abschnitts schon angedeutet, berührt sich noch ein weiteres Themenfeld der Philosophie der Raumzeit-Theorien mit unserer Thematik: der Raumzeit-Konventionalismus. Hierunter wird die These verstanden, dass die Geometrie (und gegebenenfalls auch die Topologie) der physikalischen Welt für sich genommen kein empirisches Faktum ist, sondern lediglich die Konjunktion aus Raumzeit-Geometrie und der Gesamtheit der physikalischen Gesetze. Um die Geometrie der Raumzeit empirisch zu bestimmen, benötigt man vorgängig konventionelle Annahmen über das Verhalten von Maßstäben und Uhren bei Transport. Dabei kann eine gegebene Konvention (etwa die Annahme, dass Maßstäbe bei Transport starr und von invarianter Länge sind) im Prinzip durch jede andere ersetzt werden, solange entsprechende Adjustierungen an anderer Stelle (etwa in der Optik und Elektrodynamik bezüglich des Gangs von Lichtstrahlen) vorgenommen werden. Insofern ist die Raumzeit-Geometrie für sich genommen empirisch unterbestimmt und nur die Summe aus Geometrie und Naturgesetzen Gegenstand der empirischen Prüfung.

Hacking (1975) weist nun darauf hin, dass sich durch geeignete Wahl der raumzeitlichen Struktur das PII gegen Kants Wassertropfen und Blacks Kugeln verteidigen lässt. Blacks Welt lässt eigentlich nur folgende Beschreibung zu: Gegeben ist eine Kugel mit intrinsischen Eigenschaften Q, von der aus eine Kugel mit Eigenschaften Q nach $z$ Kugeldurchmessern entlang einer geraden Linie erreichbar ist. Dies Szenario lässt sich nun entweder beschreiben durch eine Welt, die zwei Kugeln im euklidischen Raum enthält, oder aber eine Welt, die eine Kugel ent-

${ }^{11}$ Zur Einführung in die Philosophie der Raumzeit-Theorien vgl. Lyre (2007). 
hält in einem zylindrischen Raum mit Zylinderumfang $z$ (vgl. Adams, 1979, 15). Blacks Szenario zweier Kugeln lässt sich also konventionalistisch umdeuten in ein Szenario mit nur einer einzigen, leibniz-individuierten Kugel. Hacking behauptet, dass sich jeder Einwand gegen das PII konventionalistisch umdeuten lässt, so dass das PII erfüllt ist.

Für das Folgende sei von konventionalistischen Vorbehaltsklauseln abgesehen (siehe French 1975 für eine explizite Kritik an Hacking). Blacks Gedankenexperiment unterminiert dann das PII als metaphysisches Grundprinzip zur Definition von Identität. Man mag einräumen, dass es sich hierbei ja nur um eine denkbare, nicht aber um die aktuale Welt handelt. Doch selbst wenn es wahr wäre, dass sich in unser aktualen Welt keine Dinge finden lassen, die alle intrinsischen Eigenschaften teilen (was gerade der springende Punkt des Ununterscheidbarkeits-Postulats der Quantentheorie ist, wie wir im nächsten Abschnitt sehen werden), so bedroht bereits die bloße Vorstellbarkeit eines solchen Szenarios den Anspruch auf das PII als metaphysisches Grundprinzip. Eine Welt, in der ein metaphysisches Grundprinzip verletzt ist, sollte gar nicht widerspruchsfrei denkbar sein, sollte also keine logisch mögliche Welt sein. Blacks Kugeln sind aber sehr wohl vorstellbar und denkbar und konstituieren somit einwandfrei eine logisch mögliche Welt.

\section{Bündelontologie, Tropenontologie und Haecceitismus}

Doch nicht nur das Leibnizsche PII, sondern mit ihm ein ganzer, prominenter Entwurf einer Objektontologie steht auf dem Spiel: die Bündelontologie, da diese nämlich auf das PII festgelegt zu sein scheint. Der Bündelontologie zufolge sind Objekte nichts als Bündel von Eigenschaften. Zur Individuation eines Objekts als Eigenschaftsbündel steht dem Vertreter dieser Position lediglich ein Rückgriff auf eben diese Eigenschaften zur Verfügung. Dabei sollte ausgeschlossen werden, dass der Bündelontologe über Eigenschafen wie „a ist mit sich selbst identisch“ quantifiziert, denn hier ist das Beweisziel der Individuation via PII schon in die Eigenschaftsdefinition eingeflossen.

Nun sind wir bisher von Eigenschaften als Universalien ausgegangen. Wie in Fußnote 6 vermerkt, ist dies aber nicht zwingend. Eine prominente Gegenposition entwirft Eigenschaften als partikularisiert und individuiert, in der Ontologie hat sich hierfür die Bezeichnung Tropen etabliert (im Singular: die Trope). Die Vertreter einer Tropenontologie versuchen mit ihrer Position einem Charakteristikum von Eigenschafts-Unversalien zu entgehen, das insbesondere von Nominalisten als obskur angesehen wird: nämlich deren Abstraktheit bzw., im Falle instantiierter Universalien, deren multipler Lokalisiertheit. Doch Tropen sind nicht nur partikular, sondern auch numerisch distinkt, also im ontologisch primitiven Sinne bereits individuiert. Tropen sind Eigenschaftsindividuen. Demnach hat kein Elektron dieselbe Ladungstrope wie ein anderes Elektron, sondern lediglich exakt die gleiche. 
Da Vertreter der Tropenontologie typischerweise zugleich eine Bündeltheorie vertreten, konzipieren sie Dinge als Bündel von Tropen. Das Leibniz-Prinzip ist dabei trivialerweise erfüllt: da Tropen Eigenschaftsindividuen sind, besitzen keine zwei Dinge diesselben Tropen.

Einige manifeste Vor- und Nachteile von Tropen- und Universalienontologie stehen einander direkt gegenüber. Im Rahmen der Tropenontologie ist von Nachteil, dass beispielsweise die Tatsache, dass jedes Elektron im Universum exakt die gleiche Ladung trägt wie jedes andere, als nicht weiter erklärbar, also als ontologisches factum brutum angesehen werden muss. Der Vorteil der Universalienontologie liegt im Gegenzug darin, eine scheinbar natürliche Erklärung für eben dieses Faktum durch Teilhabe jedes Elektrons an derselben Ladungsuniversalie zu bieten. Den Kaufpreis hierfür zahlt die Universalientheorie durch die Annahme abstrakter Entitäten mit ominösen Teilhabe- oder Instantiierungsbedingungen.

Wie wir gesehen haben, lassen sich beide Eigenschaftskonzeptionen mit einer Bündelauffassung verknüpfen. Ein konkretes physikalisches Ding, ein Partikular, ist dann einfach das Bündel seiner Eigenschaften. Für Vertreter der Tropentheorie ist diese Option geradezu zwingend, da jede einzelne Trope bereits als ein Partikular ansehbar ist. Grundsätzlich muss die Bündelauffassung dann die Tatsache, dass an einem Raumzeitpunkt (oder in einer sehr kleinen, kompakten Raumzeitregion) zahlreiche Eigenschaften offenbar immer ko-präsent und ko-lokalisiert sind, um ein Objektbündel zu konstituieren, als factum brutum ansehen (etwa, im Falle der Tropenbündeltheorie, das Vorhandensein der Elementarladungstrope, der Spin- $\frac{1}{2}$ Trope und der Elektronenmassen-Trope am Ort jedes Elektrons). Dies ist einer der Gründe, weshalb in der Ontologie die Partikularität - und mit ihr zugleich die Identität wenigstens im Sinne numerischer Distinktheit - von der Gegenposition der Bündeltheorie, der Substanz- oder Substrattheorie, auf das Vorhandensein eines eigenschaftslosen Trägers zurückgeführt wird (der die Eigenschaften eines konkreten Objekts „trägt“ und zusammenhält). In der philosophischen Tradition sind zahlreiche Begriffe geprägt worden, um diese Position zu adressieren. Sie wird gelegentlich als Haecceitismus bezeichnet. Der Ausdruck haecceitas (lat. haec: dies) lässt sich mit „Diesheit“ übersetzen und geht auf Duns Scotus zurück. Andere Ausdrücke lauten: Lockesche Substanz (vgl. French, 1989), primitive Diesheit oder primitive Identität (Adams, 1979) transzendentale Individualität (Post, 1963) oder einfach nacktes Partikular.

Anhand der Blackschen Kugeln lässt sich die Idee illustrieren. Da die Kugeln keinerlei intrinsische Unterschiede aufweisen, aber dennoch zwei anstelle einer Kugel sind, scheinen sie ihre Identität solo numero zu besitzen: wir können eine Vielheit bzw. Kardinalität zuweisen, auch wenn die Kugeln nicht individuell zählbar sind, ihnen also keine Ordinalität zukommt. Ein weiteres, gängiges Beispiel sind die Punkte einer Raumzeit-Mannigfaltigkeit. Als Mannigfaltigkeits-Substantialismus bezeichnet man diejenige Position des Raumzeit-Substantialismus, nach der die ontologischen Konstituenten der Raumzeit Punkte der Raumzeit-Mannigfaltigkeit 
sind. Als Punkte kommen ihnen keinerlei Eigenschaften zu, sie sind völlig homogen. $\mathrm{Zu}$ Ihrer Individuation bieten Mannigfaltigkeits-Substantialisten daher an, Raumzeit-Punkte als haecceistische Entitäten anzusehen 12

Der Haecceitismus stellt die dritte Option im Rahmen der obigen Dreierliste (Seite 90 dar. Individuation im Sinne von (1) und (2) war ja, wie wir gesehen haben, mit Problemen behaftet. In beiden Fällen wird versucht, einen reduktiven Zugang anzugeben und Identität auf andere Größen zurückzuführen wie etwa Mengen von Eigenschaften oder Raumzeit-Verhalten. Individuation im Sinne von (3) sieht Identität als ontologisch irreduzibel und primitiv an. Für den Haecceitisten macht es durchaus Sinn zu fragen, ob ein bestimmtes Individuum in einer anderen möglichen Welt existiert, ohne dabei Bezug zu nehmen auf Eigenschaften oder raumzeitliches Verhalten, ohne also Individualität auf (1) oder (2) zu reduzieren. Haecceistische Unterschiede zwischen möglichen Welten sind folglich Unterschiede, die nicht auf Unterschieden von Eigenschaften oder Verhalten beruhen. Aber genau dies lässt den Haecceitismus für jede empirisch orientierte Metaphysik obskur erscheinen. Das Konzept der Identität hat, wie es scheint, so oder so seinen Preis 13

\subsubsection{Leibniz-Prinzip und Quantentheorie}

Betrachten wir noch einmal das obige Beispiel der Individuation zweier Teilchen $a$ und $b$ mit Raumzeit-Trajektorien $\gamma_{a}$ und $\gamma_{b}$. Wir wollen nun zusätzlich annehmen, dass das Raumzeitverhalten der Teilchen chaotisch ist, dass die Trajektorien $\gamma_{a}$ und $\gamma_{b}$ also praktisch unvorhersagbar sind. Falls die Teilchenorte einem Beobachter nur in diskreten Zeitintervallen zugänglich sind, besteht offenbar keine Chance einer Wiedererkennung von $a$ und $b$. Zahlreiche prominente Interpretationen der Quantentheorie, allen voran die Kopenhagener Interpretation, lehnen die Existenz

\footnotetext{
${ }^{12}$ Gerade hiergegen hatte sich Leibniz in seiner bekannten Debatte mit Clarke (respektive Newton) unter Berufung auf das PII und, vorgängig, den Satz vom zureichenden Grunde gewandt (vgl. nochmals Lyre 2007).

${ }^{13} \mathrm{Im}$ Lichte der Tropenontologie zeigt sich, dass neben Eigenschaftsindividuation im Sinne von (1), wobei Eigenschaften als Universalien zu verstehen sind, und haecceistischer Individuation im Sinne von (3) noch die Individuation mittels Tropen als weitere Option hinzutritt. Offenbar stellt sie eine Art Kombination aus (1) und (3) dar, insofern Tropen einerseits Eigenschaften sind, andererseits aber irreduzibel und primitiv. Erstaunlicherweise wird diese Option in der Debatte um Quantenidentität und Leibniz-Prinzip nirgends explizit in der Literatur betrachtet. Der Grund ist wohl, dass Unterschiede gleichartiger Tropen (also beispielsweise die Elementarladungs-Tropen zweier Elektronen) keine empirischen, sondern lediglich metaphysische Unterschiede darstellen, ähnlich wie Haecceitäten.
} 
definiter Raumzeit-Trajektorien ab ${ }^{14}$ Für Quantenobjekte scheint daher weder die Möglichkeit einer Individuation im Sinne von (1) noch von (2) zu bestehen.

Ein derartiges Argumentationsschema findet sich bis heute in zahlreichen Quantenmechanik-Lehrbüchern, beispielsweise schreiben so einflussreiche Autoren wie Landau und Lifschitz:

In der klassischen Mechanik verlieren gleichartige Teilchen (sagen wir Elektronen) trotz der Identität ihrer physikalischen Eigenschaften ihre „Individualität" nicht. [...]

In der Quantenmechanik ist die Sachlage ganz anders. Wir haben bereits mehrfach darauf hingewiesen, dass der Begriff der Bahnkurve eines Elektrons wegen des Unbestimmtheitsprinzips seinen Sinn vollkommen verliert. [...] Lokalisieren wir die Elektronen und nummerieren sie in einem gewissen Zeitpunkt durch, so haben wir dadurch nichts für ihre Identifizierung in späteren Zeitpunkten gewonnen. Wenn wir eines der Elektronen in einem anderen Zeitpunkt an einer Stelle des Raumes lokalisieren, dann können wir nicht angeben, welches der Elektronen an diesen Punkt gelangt ist.

In der Quantenmechanik gibt es also prinzipiell keine Möglichkeit, ein einzelnes von gleichartigen Teilchen gesondert zu verfolgen und damit die Teilchen zu unterscheiden. Man kann sagen, dass gleichartige Teilchen ihre „Individualität“ in der Quantenmechanik vollkommen verlieren. (Landau und Lifschitz, 1979, 218)

Entscheidend ist, dass der argumentative Fokus hier nicht auf der Frage der gleichzeitigen Unterscheidbarkeit gleichartiger Teilchen liegt, sondern auf deren Wiedererkennbarkeit in der Zeit. Man kann in systematischer Hinsicht zwischen synchroner Identität (zu einem bestimmten Zeitpunkt) und diachroner Identität, also der Persistenz eines Objekts in der Zeit, unterscheiden. Diese Unterscheidung liegt auch tentativ der Alternative einer Individuation im Sinne von (1) gegenüber (2) zugrunde. Die Problematik der Wiedererkennbarkeit wurde von den Gründervätern der Quantentheorie frühzeitig erkannt, einen expliziten Zusammenhang zum Leibniz-Prinzip stellte insbesondere Hermann Weyl her. Seine Ausführungen geben vordergründig Rätsel auf, hierüber stolpern auch Muller und Saunders (2008). Spürt man dem nach, so zeigt sich, wie sich die Sichtweise auf das Leibniz-Prinzip

\footnotetext{
${ }^{14}$ In der Bohmschen Theorie liegen die Dinge anders, wie Brown et al. (1999) zeigen. Allerdings muss hier die Möglichkeit der Beibehaltung der Individuation im Sinne von (2) dadurch erkauft werden, dass die Topologie einander nicht überschneidender Raumzeit-Trajektorien direkt in die Struktur des Konfigurationsraums und die Bohmsche Führungsgleichung eingebaut wird. In gewisser Weise ist dabei die Forderung der Impenetrabilität ontologisch primitiv.
} 
im Zusammenhang mit der Quantenmechanik historisch verändert hat. In „Gruppentheorie und Quantenmechanik" von 1928 schreibt Weyl:

...die Möglichkeit, dass eines der beiden Individuen Hans und Karl im Quantenzustand $E_{1}$, das andere im Quantenzustand $E_{2}$ sich befindet, vereinigt nicht zwei unterscheidbare Fälle, die durch die Vertauschung von Hans und Karl auseinander hervorgehen; es ist unmöglich, die wesensgleichen Individuen Hans und Karl, jedes für sich, in seiner dauernden Identität mit sich selbst festzuhalten. Von Elektronen kann man prinzipiell nicht den Nachweis ihres Alibi verlangen. So setzt sich in der modernen Quantentheorie das Leibnizsche Princip von der coincidentia indiscernibilium durch. (Weyl, 1928, 214)

Die Schlussfolgerung im letzten Satz erscheint zunächst unverständlich: Wenn doch Hans und Karl ununterscheidbar sind und in ihrer dauernden Identität nicht festgehalten werden können, es sich aber dennoch um zwei Individuen handelt, ist dann nicht das Leibnizsche Prinzip verletzt? Schaut man auf spätere Schriften, so scheint die Verwirrung sogar noch zuzunehmen, denn 1949 schreibt Weyl:

Das Ende von alledem ist, dass die Elektronen Leibnizens principium identitatis indiscernibilium befriedigen, oder dass das Elektronengas ein „monomisches Aggregat“ ist (Dirac-Fermi-Statistik). In einem tiefen und präzisen Sinn bestätigt die Physik die Mutakallimūn: weder dem Photon, noch dem (positiven und negativen) Elektron kann man Individualität zuschreiben. Es hat sich herausgestellt, dass das Leibniz-Pauli-Verbot für Elektronen, doch nicht für Photonen gilt. (Weyl, 1966, 316-317)

Wiederum ist von einer Bestätigung des Leibniz-Prinzips die Rede, diesmal allerdings mit Hinweis darauf, dass das Elektronengas (und aufgrund der vorhergehenden Passagen im gleichen Sinne wohl auch das Photonengas) ein „monomisches Aggregat", also ein Ganzes, bildet. Bemerkenswert ist ferner Weyls Redeweise vom „Leibniz-Pauli-Verbot“ (hierauf soll weiter unten eingegangen werden).

Den Passagen kann aber sehr wohl eine stimmige Lesart abgewonnen werden. Weyl hat offenbar nicht das Blacksche Szenario (als Gegenbeispiel des PII) vor Augen, er betrachtet vielmehr, ähnlich wie später Landau und Lifschitz, die Frage der Wiedererkennbarkeit in der Zeit, diskutiert also die diachrone im Gegensatz zur synchronen Identität. Nun sind die zwei Szenarien von Hans und Karl vor und nach der Vertauschung empirisch ununterscheidbar, sie müssen daher physikalisch als ein einziges Szenario gezählt werden - im Sinne des PII.

Die numerische Distinktheit der Elektronen zu einem Zeitpunkt, also deren synchrone Identität, stellt Weyl erst gar nicht in Frage. Vielleicht hat er stillschweigend eine haecceistische Position vertreten, dies bleibt in den genannten Passagen offen. In seinem späteren Buch „Symmetry“ von 1952 schreibt er: 
I told you that Leibniz had given the geometric notion of similarity this philosophical twist: Similar, he said, are two things which are indiscernible when each is considered by itself. Thus two squares in the same plane may show many differences when one regards their relation to each other; for instance, the sides of the one may be inclined by $34^{\circ}$ against the sides of the other. But if each is taken by itself, any objective statement made about one will hold for the other; in this sense they are indiscernible and hence similar. (Weyl, 1952, 127-128)

Abermals: Die zwei Quadrate können zwar relational in ihrer Zweiheit unterschieden werden, im Sinne des ,Quadratseins' sind sie aber gleichartig, also ununterscheidbar. Wäre Weyl der Frage der relationalen Unterscheidung der Quadrate weiter nachgegangen, hätte er den Fokus also auf synchrone Identität verschoben, so wäre er womöglich bei Überlegungen ausgekommen, die den späteren Debatten wie in Abschnitt 3.2 .3 beschrieben nahe kommen. Statt dessen finder sich in der frühen Debatte um Quantenidentität die Betonung eines holistischen Aspekts, der darin besteht, dass Vielheiten gleichartiger Quantenojekte „monomische“ Ganze bilden in dem Sinne, dass zwischen dem Zustand $\mid$ Hans $\rangle+\mid$ Karl $\rangle$ und dessen Permutation $\mid$ Karl $\rangle+\mid$ Hans $\rangle$ physikalisch nicht unterschieden werden kann, und sie daher nach Leibniz ein Ganzes bilden. Entsprechend verbietet sich die physikalisch sinnvolle Rede über die (individuellen) Teile eines derartigen Ganzen. Max Born schreibt 1927, dass Teilchen ,in vielen Fällen gar nicht als Individuen zu identifizieren [sind], z.B. dann, wenn sie zu einem Atomverband zusammentreten“ (Born, 1927, 240). Ernst Cassirer greift dies 1937 auf und schreibt

Die Unmöglichkeit, verschiedene Elektronen gegeneinander abzugrenzen und ihnen je eine selbstständige „Individualität“ zuzuschreiben, ist in der Entwicklung der neueren Quantentheorie vor allem durch die Erwägungen, die sich an das „Pauli-Verbot" angeknüpft haben, in helles Licht gerückt worden. Betrachtet man das Paulische Äquivalenzprinzip lediglich nach der methodischen Bedeutung ..., so tritt eine eigentümliche Analogie zwischen ihm und [dem Leibniz-Prinzip...] hervor [...] Das Pauli-Prinzip ist gewissermaßen das „Principium identitatis indiscernibilium“ der Quantentheorie. Es charakterisiert jedes Elektron innerhalb des Atoms durch einen bestimmten Inbegriff von Bedingungen: durch die vier „Quantenzahlen“ ...- und es spricht weiterhin die Folgerung aus, dass Elektronen, die im Hinblick auf diese Charakteristik keine Unterschiede aufweisen, als ein einziges physikalisches Sein anzusehen sind. (Cassirer, 1937, 341, Fußnote 47)

Ebenso wie Weyl analogisiert Cassirer die Prinzipien von Leibniz und Pauli. Nach dem Pauli-Prinzip besitzen keine zwei Fermionen die gleichen Quantenzahlen, also Eigenschaften. Dies sieht nach einer Analogie zum PII aus - und zwar 
auch im Sinne synchroner Identität. Da ein derartiges Verbot für Bosonen aber nicht besteht, sollte hier eine Unterscheidung erfolgen. Die Passagen sowohl von Weyl als auch von Cassierer sind in dieser Hinsicht unbefriedigend. Dies hat nicht nur mit der Unterscheidung von Fermionen und Bosonen zu tun, sondern eben damit, ob man das Leibniz-Prinzip zur diachronen oder zur synchronen Individuation heranzieht. Letzteres kommt im weiteren Verlauf der Debatte immer mehr in den Fokus mit der Folge, dass nun zum Teil gegenteilige Konsequenzen gezogen werden (zumindest dem Wortlaut nach).

Henry Margenau spricht 1944 explizit von einem Widerspruch zwischen PauliPrinzip und Leibniz-Prinzip und einer Verletzung des letzteren. Er sagt zunächst:

This conclusion recalls Leibnitz' principle of the identity of indiscernibles; indeed physicists have occasionally thought that the E.P. [exclusion principle] implies this principle with regard to elementary particles of the same species.

Leider lässt Margenau offen, welche Physiker es waren, die dies zunächst behauptet haben (aber es liegt natürlich nahe, dass er hierbei auch an Weyl dachte). Dann fährt er fort:

... the E.P., so far as it goes, contradicts Leibnitz [...] two particles, as we have seen, differ in no observable respect. Nevertheless quantum mechanics would lead to entirely erroneous results if they were treated as a single entity. The particles, though they can not be labelled individually, can be counted. If and only if identity were understood as not implying numerical identity, then two electrons in an atom could be said to be identical. (Margenau, 1944, 202)

Hier ist nun in klarer Weise die synchrone Fragestellung adressiert und damit die Frage, wie es denn sein kann, dass zwei Teilchen, obwohl sie „in no observable respect" differieren, also empirisch ununterscheidbar sind, dennoch zwei Teilchen sind. Dies lässt scheinbar nur einen Schluss zu: in synchroner Hinsicht ist das PII verletzt.

Genau diese Ansicht findet, vor allem vonseiten der Wissenschaftsphilosophie, in der Folge zunehmend Anhänger (siehe z.B. Post 1963, Cortes 1976, Teller 1983. French und Redhead 1988 und Butterfield 1993). Castellani und Mittelstaedt schreiben: „[I]t is also commonly held that a form of the principle of the identity of indiscernibles is valid in the domain of classical physics, while the principle is inapplicable in the quantum case" und sie setzen in Fußnote hinzu: "This is undoubtly the prevailing position in the literature" (Castellani und Mittelstaedt, 2000, 1589). Insofern ist Steven French durchaus zuzustimmen, wenn er die Verletzung des Leibniz-Prinzips in der Quantentheorie in seinem Enzyklopädieartikel über „Identity and Individuality in Quantum Theory“ (French, 2011) als „received view", als die herrschende Schulmeinung, bezeichnet. 
Wenn aber das PII in der Quantentheorie verletzt ist, was folgt dann daraus? Die bisherigen Ausführungen machen deutlich, dass hier eine Reihe von Fragen auseinandergehalten werden müssen, unter anderem:

1. In welchem Sinne könnten Quantenobjekte (keine) Individuen sein?

2. Verletzen Quantenobjekte das Leibniz-Prinzip?

3. Gibt es einen ontologischen Unterschied zwischen Fermionen und Bosonen?

4. In welchem Sinne besteht einen Vielteilchenzustand de facto aus vielen Teilchen (oder muss er nicht vielmehr als ein Ganzes angesehen werden)?

Die Motive für diese Fragen tauchten im Verwirrspiel der obigen Zitate bereits verschiedentlich auf. Sie sind im Folgenden noch genauer zu behandeln. Im Zusammenhang mit der dritten Frage kehren wir im nächsten Abschnitt 3.2.3 zur zweiten Frage zurück, die hier insofern nur vorläufig bejaht wird. Die vierte Frage wird in Abschnitt 3.2.4 aufgegriffen; wenden wir uns zunächst der ersten Frage zu.

Haben wir es bei Quantenobjekten mit Nicht-Individuen zu tun? Was sollte das bedeuten? In der Tat scheint die Quantentheorie das Blacksche Szenario Wirklichkeit werden zu lassen: gleichartige Quantenobjekte besitzen eine Kardinalität, keine Ordinalität. Dies ist eine direkte Konsequenz des UnunterscheidbarkeitsPostulats - gleichartige Quantenobjekte befinden sich in Zuständen, für die es möglich ist, eine Objekt-Gesamtzahl anzugeben, obwohl die Objekte für sich empirisch ununterscheidbar sind. Man hat versucht, dies in didaktische Bilder zu kleiden: Die Geldmenge in meinem Portemonaie ist durch die einzelnen Münzen leibniz-individuiert, derselbe Geldbetrag auf meinem Konto ist es nicht ${ }^{15}$ Es bleibt zunächst unklar, ob dies eine zulässige Analogie ist, ob wir mathematische Verhältnisse auf die Welt abbilden dürfen (siehe hierzu 3.2.4 und ob wir dadurch das Wesen von Kardinalität erfasst haben. Quantenobjekte scheinen eine Art Verschiedenheit solo numero zu besitzen - im Widerspruch zum Leibniz-Prinzip. Folgt daraus dann nicht, dass die Quantentheorie den Haecceitismus bestätigt? Besitzen Quantenobjekte also eine primitive Identität, die sich weder an der raumzeitlichen Historie noch an Eigenschaften festmachen lässt? Interessanterweise haben nur wenige Autoren diese Konsequenz explizit gezogen, und dies, obwohl die Überzeugung, dass die Quantenmechanik das PII verletzt, sich als vorherrschend durchsetz-

\footnotetext{
${ }^{15}$ Schrödinger benutzt diese Illustration 1949: „,... the shillings and pennies in your bank account are not individuals." (zitiert nach French und Krause, 2006, 122), und Mary Hesse schreibt:
}

With pounds, shillings, and pence in a bank balance, however, it is not merely the case that we cannot in practice re-identify a given pound appearing in the credit column, but that there is no sense in speaking of the self-identity of this pound, and of asking where it reappears in another column or whether it is the pound paid over the counter yesterday. (Hesse, 1966, 49-50) 
te (vgl. French und Redhead, 1988). In jüngerer Zeit bevorzugen einige Autoren sogar eine Revision der Logik oder Mengenlehre (siehe 3.2.4.

\subsubsection{Schwache Unterscheidbarkeit}

Sowohl Weyl als auch Cassirer haben die Prinzipien von Leibniz und Pauli analogisiert. Pauli selbst war hiermit keineswegs einverstanden, 1949 schreibt er an Fierz sinngemäß, dass das Leibniz-Prinzip als metaphysisches Prinzip doch eigentlich keine empirischen Konsequenzen haben kann, und dann wörtlich:

Das wäre doch ein merkwürdiges Prinzip in der Philosophie von Leibniz, das nicht für alle Objekte gilt (z.B. nicht für Photonen, was Weyl ausdrücklich betont), sondern nur für manche ${ }^{16}$

Auch Weyl wird von Pauli mit einem Brief bedacht. Zwar zeigen die Briefe, dass Pauli über das PII nicht genau informiert war, aber er berührt die oben bereits angesprochene, wichtige Frage, ob zwischen Bosonen und Fermionen hinsichtlich PII und Ontologie ein Unterschied besteht. Dieser Punkt verdient eine genauere Betrachtung.

Das Pauli-Prinzip besagt ja, dass sich keine zwei Fermionen im gleichen Zustand befinden können, dass sie sich also in wenigstens einer Quantenzahl, also einer Eigenschaft, unterscheiden müssen. Insofern entsteht in der Tat der Eindruck, dass das Leibniz-Prinzip von Fermionen erfüllt wird. Wieso kommen dann aber Margenau und der „received view“ zur gegenteiligen Ansicht? Die Anwendung eines Permutationsoperators auf einen Fermionenzustand (3.6) führt ja lediglich auf einen Vorzeichenwechsel, darüber hinaus entspricht seine Wirkung derjenigen auf einen Bosonenzustand 3.4. Da ferner jede physikalische Observable $\hat{O}$ mit jedem Permutationsoperator $\hat{P}$ kommutiert (3.5), sind die Erwartungswerte aller Operatoren für ein einzelnes Fermion in einem Viel-Fermionen-Zustand dieselben. In dieser Hinsicht besteht kein Unterschied zwischen Fermionen und Bosonen: Durch keine physikalische Messung kann eine Unterscheidung zwischen einzelnen Fermionen oder Bosonen vorgenommen werden.

Trotz dieser zunächst überzeugenden Argumentation des „received view“ hat die Debatte um den Status des Leibniz-Prinzips in der Quantentheorie in der ersten Hälfte der 2000er Jahre völlig unerwartet neuen Schub bekommen. Einen entscheidenden Anstoß hierzu lieferten die Arbeiten von Simon Saunders (2003, 2006). Saunders greift dabei auf frühere Arbeiten von Quine über schwache Unterscheidbarkeit zurück - und deckt eine Ungenauigkeit in unserer bisherigen Argumen-

\footnotetext{
${ }^{16}$ Zitiert nach von Meyenn 1987, siehe dort speziell den zweiten Abschnitt zum AusschlieBungsprinzip und zur Teilchenunterscheidbarkeit.
} 
tation auf. Wir waren bislang davon ausgegangen, dass im PII über monadische, intrinsische Eigenschaften quantifiziert wird. Dies ist aber unter Umständen eine unnötig starke Forderung. Gibt man diese Forderung auf, so lassen sich LeibnizPrinzipien unterschiedlicher Stärkegrade formulieren, je nachdem, über welche Arten von Eigenschaften quantifiziert wird. Neben intrinsischen Eigenschaften lassen sich auch relationale Eigenschaften betrachten, wobei insbesondere Ordungsrelationen und irreflexive Relationen zu interessanten Erweiterungen des Konzepts der Unterscheidbarkeit führen.

Nach Quine 1976) lassen sich drei Arten von Unterscheidbarkeit differenzieren: absolute, relative und schwache Unterscheidbarkeit. Sie sind wie folgt definiert:

- Absolut unterscheidbare Objekte sind in wenigstens einer monadischen Eigenschaft verschieden.

- Relativ unterscheidbare Objekte sind hinsichtlich wenigstens einer Ordnungsrelation verschieden.

- Schwach unterscheidbare Objekte sind hinsichtlich wenigstens einer irreflexiven Relation verschieden.

Einige paradigmatische Beispiele: Natürliche Zahlen sind absolut unterscheidbar. Demgegenüber sind die Zeitpunkte des Zeitpfeils zwar intrinsisch gleich, insofern nicht absolut unterscheidbar, jedoch relativ unterscheidbar hinsichtlich der Früherspäter-Relation. Schwach unterscheidbare Objekte bedürfen irreflexiver Relationen, deren Definition hier zunächst angegeben werden soll:

Eine Relation $R$ ist reflexiv, wenn für alle $x$ in der betrachteten Domäne $R(x, x)$ gilt. Falls $\neg R(x, x)$ gilt, ist $R$ irreflexiv.

Blacksche Kugeln im Abstand $d$ statuieren eine irreflexive Abstandsrelation: jede Blacksche Kugel ist von der anderen um $d$ entfernt, aber nicht von sich selbst.

Die verschiedenen Formen von Unterscheidbarkeit lassen sich auch anhand von Graphen illustrieren: Im einfachen Fall eines benannten Graphen („labelled graph") mit zwei Knoten und einer Kante

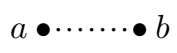

sind die beiden Knoten absolut unterscheidbar. Hierbei kann „....." sowohl eine gerichtete als auch ungerichtete Verbindung sein. Der unbenannte und gerichtete Graph

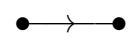

stellt ein Beispiel für relative Unterscheidbarkeit der Knoten dar, der unbenannte und ungerichtete Graph 
ein Beispiel für schwach unterscheidbare Knoten. Demgegenüber sind die beiden Knoten des kantenlosen Graphen

nicht einmal schwach individuierbar.

Mit den drei obigen Varianten von Unterscheidbarkeit ergeben sich nun drei Varianten des Leibniz-Prinzips(in unterschiedlichen Stärkegraden; jeweils formuliert in der bereits oben favorisierten, kontrapositiven Form):

Starkes PII: Es existieren keine zwei Individuen, die nicht absolut unterscheidbar sind.

Moderates PII: Es existieren keine zwei Individuen, die nicht relativ unterscheidbar sind.

Schwaches PII: Es existieren keine zwei Individuen, die nicht schwach unterscheidbar sind.

In den vorhergehenden Abschnitten wurde das Leibniz-Prinzip im Sinne des starken PII diskutiert. Saunders (2006) und Muller und Saunders (2008) zeigen nun, dass Fermionen zwar das starke, nicht aber das schwache PII verletzen. Man betrachte etwa die antisymmetrisierte Zustandsfunktion (3.6) zweier Fermionen, im konkreten Beispiel etwa das Spin-Singulett zweier ansonsten in allen Quantenzahlen übereinstimmender Elektronen ${ }^{17}$ mit den Spineinstellungen $|\uparrow\rangle$ und $|\downarrow\rangle$

$$
|\Psi\rangle=\frac{1}{\sqrt{2}}(|\uparrow\rangle|\downarrow\rangle-|\downarrow\rangle|\uparrow\rangle) .
$$

Beide Elektronen genügen der irreflexiven Relation $R=$ „haben entgegengesetzten Spin zueinander, jedoch nicht zu sich selbst“. Die Elektronen oder allgemeiner Fermionen sind daher nach Saunders schwach unterscheidbar, ihre Identität lässt sich mit Hilfe des schwachen PII in $R$ fundieren.

\footnotetext{
${ }^{17}$ Ein derartiger Zustand stellt eine didaktische Vereinfachung dar, die zwar gang und gäbe ist, die aber zu falschen Schlussfolgerungen führen kann. Insbesondere fallen Antisymmetrie und EPR-Verschränkung nicht zusammen. Denn die vollständige Zustandsbeschreibung eines Elektrons muss ja neben den Spinfreiheitsgraden noch die Freiheitsgrade im Raum mit umfassen, der Zustand 3.16 könnte sonst so missverstanden werden, dass sich beide Spins am selben Raumzeitpunkt befinden, was im Falle zweier Elektronenspins offensichtlich unmöglich ist. Für Teilchen ist der vollständige Zustand eine Wellenfunktion im Spin-OrtsRaum. Dort sind aber Zustände, die durch direkte Antisymmetrisierung von Produktzuständen entstehen, noch nicht EPR-verschränkt (im Sinne von Kapitel 4). Antisymmetrie und EPR-Verschränkung sind konzeptionell voneinander zu trennen, wie Ghirardi et al. (2002) ausführlich zeigen.
} 
Folgt man dieser Argumentation, so sind Fermionen in derselben Weise schwach unterscheidbar wie Blacksche Kugeln, nicht aber Bosonen. Saunders hält dies für unproblematisch, denn es sind die elementaren Fermionen - Leptonen und Quarks -, die den Aufbau der Materie bestimmen, während elementare Bosonen nur als Eichteilchen und Higgs-Boson auftauchen. Als solche sind sie nach Saunders nicht als Objekte, sondern als Anregungsmoden von Quantenfeldern anzusehen: „We went wrong in thinking the excitation numbers of the mode, because differing by integers, represented a count of things; the real things are the modes" (Saunders, 2006, 60). Es ist fraglich, inwieweit eine solche Trennung von Fermionen und Bosonen ontologisch plausibel ist, mindestens ebenso fraglich ist, inwieweit Eichbosonen (in sowohl masseloser als auch massiver Form) und Higgs-Bosonen ontologisch gleichrangig zu behandeln sind, denn schließlich kommt dem Higgs nicht die Rolle eines Wechselwirkungsfeldes zu.

Doch man muss diese Fragen nicht weiter vertiefen, folgt man der Argumentation von Muller und Seevinck (2009), die zeigen, dass sich Saunders' Resultat auf Bosonen bzw. sämtliche Quantenobjekte erweitern lässt. Ihre Grundidee ist, dass Quantenobjekte in einem Mehrobjekt-Zustand aufgrund der nicht-kommutativen Algebrastruktur der Quantentheorie zwangsläufig bestimmten Heisenbergschen Kommutatorrelationen genügen müssen, etwa der irreflexiven Relation „haben komplementären Ort und Impuls zueinander" oder allgemeiner „genügen kanonisch konjugierten Variablen“. Nach dieser Argumentation spielt es keine Rolle, ob wir Fermionen oder Bosonen betrachten, sämtliche Quantenobjekte genügen dem schwachen PII (siehe auch Huggett und Norton 2014 für eine Verfeinerung der Argumente).

Die Arbeiten von Saunders und Muller zur schwachen Unterscheidbarkeit haben die Diskussion um den Status des PII und allgemein die Ontologie der Quantentheorie neu belebt, sind aber nicht unkontrovers geblieben. Dies leitet über zum letzten Abschnitt.

\subsubsection{Ausblick}

Rekapitulieren wir kurz den Stand der Dinge: Unstrittig in der Debatte um die Ontologie der Quantentheorie sind der empirische Befund der physikalischen Ununterscheidbarkeit der Anwendung einer Teilchenpermutation auf einen Vielteilchenzustand im Sinne des Ununterscheidbarkeits-Postulats sowie die formalen Konsequenzen im mathematischen Apparat in Form des Symmetrisierungs-Postulats und der Permutationsinvarianz. In der Diskussion um das Leibniz-Prinzip sahen Weyl und Cassirer es zunächst in diachroner Hinsicht bestätigt, während sich im Anschluss an Margenau durchsetzte, dass in synchroner Hinsicht das (starke) PII in der Quantentheorie verletzt ist. Die Arbeiten von Saunders und Muller rehabilitieren das Leibniz-Prinzip auch in synchroner Hinsicht, jedoch nicht in seiner 
starken Form, sondern nur als schwaches PII. Doch auch diese Schlussfolgerung bleibt nicht unwidersprochen, dies sei hier ausblickhaft dargestellt.

Der Ansatz, eine Fundierung („grounding“) von Identität oder Individualität mittels Leibniz-Prinzipien über Eigenschaften zu gewährleisten, kann als Reduktionismus angesehen werden. Identität superveniert über Eigenschaften und ist insofern eine abgeleitetes Konzept. Der Haecceitismus stellt demgegenüber einen Antireduktionismus bzw. Primitivismus von Identität dar. Im Falle schwach unterscheidbarer Objekte reduziert sich Identität auf rein relationale Eigenschaften. Da jedoch der Begriff der Relation auch denjenigen der Relata voraussetzt, droht hier nach Ansicht einiger Autoren ein Zirkel. Katherine Hawley (2009) argumentiert, dass schwache Unterscheidbarkeit in der Tatsache ihre Fundierung findet, dass die betreffenden Objekte bereits distinkt, also verschieden sind, und dass man daher nicht umgekehrt die Unterscheidbarkeit als Grundlage ihres Verschiedenseins ansehen könne - kurz: keine Relationen ohne Relata. Wie es scheint, droht ein unauflösliches Patt in der Debatte zwischen Reduktionismus und Antireduktionismus von Identität. Dorato und Morganti (2013) schlagen sogar eine pluralistische Strategie vor.

Eine andersartige Kritik an der Rehabilitation des schwachen PII in der Quantentheorie durch Saunders und Muller haben Dieks und Versteegh (2008) vorgelegt. Die Autoren verweisen auf die manifesten Unterschiede zwischen klassischer und Quantenwelt. Sie stimmen zu, dass Blacksche Kugeln schwach unterscheidbar sind, lehnen aber die Übertragbarkeit dieses Szenarios auf den Quantenfall ab. Nach ihrer Auffassung darf ein „Vielteilchenzustand“ in der Quantentheorie nicht so angesehen werden, als würde er faktisch bereits aus Einzelobjekten bestehen sondern allenfalls potenziell zufolge möglicher, dann aber faktisch durchzuführender Messungen. In den Worten der Autoren:

There is no sign within the standard interpretation of quantum mechanics that „identical fermions" are things at all; there is no ground for the supposition that the quantum relations "between fermions" connect any actual physical objects. The irreflexivity of these relations does not help us here. Quantum relations have a standard interpretation not in terms of what is actual, but rather via what could happen in case of a measurement (Dieks und Versteegh, 2008, 934).

Der Verteidiger des schwachen Quanten-PII könnte im Gegenzug darauf verweisen, dass die Frage bestehen bleibt, wieso es quantentheoretisch möglich ist, einem Vielteilchenzustand eine Teilchenzahl zuzuweisen, wenn doch die Teile nicht aktual bestehen (siehe auch Ladyman und Bigaj 2010 für eine Reaktion auf Dieks). Andererseits scheint es umgekehrt merkwürdig, dass der Unterschied zwischen klassischer und Quantenphysik bei Fragen der Individuation keine Rolle spielen soll. 
Ferner berührt sich die Debatte um die Quantenontologie an wenigstens vier Stellen in bemerkenswerter Weise mit der in jüngerer Zeit florierenden Debatte um den Strukturenrealismus, einer moderaten Variante eines wissenschaftlichen Realismus mit vorrangigem Bezug auf die moderne Physik. Strukturenrealisten, vor allem in der weit verbreiteten ontischen Spielart dieser Position, vertreten die Ansicht, dass die fundamentalen Entitäten der Welt strukturell individuiert sind. Wie dies genau zu verstehen ist, ist Gegenstand eigener Diskussionen und kann im Folgenden nur implizit deutlich gemacht werden (vgl. Lyre 2012 und French 2014 als umfassende Darstellung). Steven French (1989) argumentiert, dass die Quantentheorie sowohl mit der Annahme verträglich ist, Quantenobjekte seien keine Individuen (im Leibnizschen Sinne aufgrund der Verletzung des starken PII), als auch mit der gegenteiligen Annahme, sie seien Individuen (im haecceistischen Sinne). Wir haben es also mit einer Unterbestimmtheit in Bezug auf die Metaphysik selbst zu tun. Diese metaphysische Unterbestimmtheit ist nach French und Ladyman (2003) ein Beleg dafür, dass eine an objekt-artigen Entitäten orientierte Ontologie fehl geht und durch eine strukturelle Metaphysik zu ersetzen ist.

French und Krause (2006) gehen noch weiter und versuchen, eine revidierte Mengenlehre für Quasi-Objekte zu entwickeln. Hierin besteht eine zweite Berührstelle zwischen Quantenontologie und Strukturenrealismus. Eine dritte Berührstelle hängt mit der Beobachtung zusammen, dass die Permutations-Invarianz der Quantentheorie eine Entsprechung in der Allgemeinen Relativitätstheorie in Form der Diffeomorphismen-Invarianz hat, genauer: Nach John Stachel (2002) zielen beide Invarianzen auf die abstrakte Eigenschaft bestimmter Theorien, allgemein permutierbar zu sein. Eine Theorie $\mathrm{T}$ ist allgemein permutierbar, falls Modelle von $\mathrm{T}$ als äquivalent angesehen werden, die sich nur darin unterscheiden, welche Objekte welche Stellen oder Rollen in einem Netz von Relationen einnehmen. In der Allgemeinen Relativitätstheorie bezieht sich die Diffeomorphismen-Invarianz auf die allgemeine Permutierbarkeit von Raumzeit-Punkten, für die Quantentheorie argumentieren Caulton und Butterfield (2012), dass sie allgemein permutierbar ist, wenn man die volle Symmetrische Gruppe, also auch die gemischt-symmetrischen Darstellungen bzw. Parastatistik mit betrachtet. Ob derartige Objekte in der Natur faktisch realisiert sind, ist sekundär; entscheidend ist, dass Ununterscheidbarkeitsund Symmetrisierungspostulat der Quantentheorie die Möglichkeit hierzu beinhalten.

Viertens ist das schwache PII vordergründig durchaus verträglich mit einem Strukturenrealismus: Objekte oder Relata werden lediglich bis auf irreflexive Relationen individuiert. Die weiter oben schon artikulierte Kritik an nicht in Relata fundierten Relationen in Zusammenhang mit dem schwachen PII kann von strukturalistischer Seite auch so gewendet werden, dass entweder Relationen und ihre Relata ontologisch auf derselben Stufe stehen, oder aber die Relata bloße Knotenpunkte in einem Netz aus Relationen sind. Strukturalismus (sowohl in Physik als auch Mathematik) ist in allgemeinster Hinsicht gerade definierbar als diejeni- 
ge Position, die die Individualität oder Kardinalität (numerische Verschiedenheit) von Objekten in deren Verortung oder Rolle in einem Gefüge oder Netzwerk relationaler Eigenschaften fundiert. Somit treffen sich die Projekte des Strukturenrealismus und der Verteidigung des schwachen PII genau bei dem Versuch, eine Fundierung von Relationen zu erreichen, die weder in einen starken Objektbegriff nach Maßgabe des starken PII mündet noch in einen Haecceitismus oder gar einen Eliminativismus (im Sinne von Relationen ohne Relata), sondern mit einer schlanken Konzeption von Objekten bzw. Relata im Sinne primitiver numerischer Verschiedenheit auskommt (,thin objects“; vgl. French und Ladyman 2011) 18

Die Debatte um die Ontologie der Quantentheorie zeigt exemplarisch, wie sich Fortschritt in der Philosophie sehr häufig gestaltet: Neue Einsichten werden gewonnen und die Diskussion auf ein höheres und abstrakteres Niveau gehoben. Doch es ergeben sich nicht minder vertrackte Anschlussfragen, und die Debatten bleiben offen. Wie offen die Debatte im Falle der Quantenontologie ist, haben diese letzten Ausblicke gezeigt.

\section{Literatur zu Kapitel 3}

Adams, Robert M. (1979). Primitive thisness and primitive identity. Journal of Philosophy $76,5-26$.

Black, Max (1952). The identity of indiscernibles. Mind 61, 153-64.

Born, Max (1927). Quantenmechanik und Statistik. Naturwissenschaften 15, 238-242.

Butterfield, Jeremy (1993). Interpretation and identity in quantum theory. Studies in History and Philosophy of Science 24, 443-476.

Caulton, Adam und Jeremy Butterfield (2012). Symmetries and paraparticles as a motivation for structuralism. British Journal for the Philosophy of Science 63(2), 233-285.

Brown, Harvey, Erik Sjöqvist und Guido Bacciagaluppi (1999). Remarks on identical particles in de Broglie-Bohm theory. Physics Letters A 251, 229-235.

Cassirer, Ernst (1937). Determinismus und Indeterminismus in der modernen Physik: historische und systematische Studien zum Kausalproblem. Göteborg: Wettergren und Kerbers Förlag.

Castellani, Elena, Hg. (1998). Interpreting Bodies: Classical and Quantum Objects in Modern Physics. Princeton: Princeton University Press.

\footnotetext{
${ }^{18}$ Nach Leitgeb und Ladyman 2008 könnte die Welt einem kantenlosen Graphen entsprechen, ohne dass dies in einen Haecceitismus mündet, sondern immer noch in Einklang mit dem Strukturalismus stünde. Graphentheoretisch genügt der kantenlose Graph 3.15 ebenso wie sein Gegenstück mit Kanten (3.14) denselben nicht-trivialen Automorphismen, beide sind strukturelle Invarianten unter Knotenpermutation. Dennoch wird die Verschiedenheit der Knoten in 3.15 durch keinerlei Relationen, nicht einmal durch schwach unterscheidende, irreflexive Relationen fundiert.
} 
Castellani, Elena und Peter Mittelstaedt (2000). Leibniz's principle, physics, and the language of physics. Foundations of Physics 30(10), 1587-1604.

Cortes, Alberto (1976). Leibniz's principle of the identity of indiscernibles: A false principle. Philosophy of Science 43(4), 491-505.

Darrigol, Oliver (1991). Statistics and combinatorics in early quantum theory II: Early symptoms of indistinguishability and holism. Historical Studies in the Physical Sciences $21,237-98$.

Della Rocca, Michael (2005). Two spheres, twenty spheres, and the identity of indiscernibles. Pacific Philosophical Quarterly 86, 480-492.

Dieks, Dennis und Marijn A. M. Versteegh (2008). Identical quantum particles and weak discernibility. Foundations of Physics 38, 923-934.

Dorato, Mauro und Matteo Morganti (2013). Grades of individuality. A pluralistic view of identity in quantum mechanics and in the sciences. Philosophical Studies 163(3), 591-610.

Feynman, Richard P., Robert B. Leighton und Matthew Sands (1964). The Feynman Lectures on Physics, Vol. 3. Reading, MA: Addison-Wesley.

French, Steven (1975). Hacking away at the identity of indiscernibles: Possible worlds and Einstein's principle of equivalence. Journal of Philosophy 92(9), 455-466.

French, Steven (1989). Identity and individuality in classical and quantum physics. Australasian Journal of Philosophy 67, 432-446.

French, Steven (2011). Identity and individuality in quantum theory. The Stanford Encyclopedia of Philosophy (Summer 2011 Edition), http://plato.stanford.edu

French, Steven (2014). The Structure of the World. Oxford: Oxford University Press.

French, Steven und James Ladyman (2003). Remodelling structural realism: Quantum physics and the metaphysics of structure. Synthese 136(1), 31-56.

French, Steven und Michael Redhead (1988). Quantum physics and the identity of indiscernibles. British Journal for the Philosophy of Science 39, 233-246.

French, Steven und Decio Krause, Hg. (2006). Identity and Individuality in Modern Physics. Oxford: Oxford University Press.

French, Steven und James Ladyman (2011). In defence of ontic structural realism. In: P. Bokulich und A. Bokulich (Hg.), Scientific Structuralism. New York: Springer.

Ghirardi, GianCarlo, Luca Marinatto und Tullio Weber (2002). Entanglement and properties of composite quantum Systems: A conceptual and mathematical analysis. Journal of Statistical Physics 108, 49-122.

Hacking, Ian (1975). The identity of indiscernibles. Journal of Philosophy 72(9), 249-256.

Hawley, Katherine (2009). Identity and indiscernibility. Mind 118(1), 101-119.

Hesse, Mary B. (1966). Models and Analogies in Science. Notre Dame, IN: University of Notre Dame Press.

Huggett, Nick und Josh Norton (2014). Weak discernibility for quanta, the right way. British Journal for the Philosophy of Science 65(1), 39-58.

Ladyman, James und Tomasz Bigaj (2010). The principle of the identity of indiscernibles and quantum mechanics. Philosophy of Science 77(1), 117-136. 
Landau, Lew und Jewgeni Lifschitz (1979). Lehrbuch der theoretischen Physik - Band III: Quantenmechanik. Berlin: Akademie-Verlag (russische Originalausgabe: Nauka, Moskau 1974).

Leitgeb, Hannes und James Ladyman (2008). Criteria of identity and structuralist ontology. Philosophia Mathematica (III) 16, 388-396.

Lewis, David K. (1986). On the Plurality of Worlds. Oxford: Blackwell.

Loux, Michael J. (1998, $\left.{ }^{3} 2006\right)$. Metaphysics: A Contemporary Introduction. London: Routledge.

Lyre, Holger (2007). Philosophische Probleme von Raumzeit-Theorien. In: A. Bartels und M. Stöckler (Hg.). Wissenschaftstheorie. Ein Studienbuch, 223-244. Paderborn: Mentis.

Lyre, Holger (2012). Symmetrien, Strukturen, Realismus. In: M. Esfeld (Hg.), Philosophie der Physik, 368-389. Berlin: Suhrkamp.

Margenau, Henry (1944). The exclusion principle and its philosophical importance. Philosophy of Science 11(4), 187-208.

Messiah, Albert (1979). Quantenmechanik, Band 2. Berlin: W. de Gruyter (franz. Original: Mécanique quantique, Paris, 1959).

Messiah, Albert und Oscar Greenberg (1964). Symmetrization postulate and its experimental foundation. Physical Review 136(1B), 248-267.

Muller, Fred A. und Simon Saunders (2008). Discerning fermions. British Journal for the Philosophy of Science 59, 499-548.

Muller, Fred A. und Michiel P. Seevinck (2009). Discerning elementary particles. Philosophy of Science 76, 179-200.

Meyenn, Karl von (1987). Pauli's belief in exact symmetries. In: M. Doncel (Hg.), Symmetries in Physics (1600-1980), 329-358. Barcelona: Universitat Autònoma de Barcelona.

Post, Heinz (1963). Individuality and physics. The Listener 70, 534-537.

Quine, Willard van Orman (1969). Ontological Relativity and Other Essays. New York: Columbia University Press.

Quine, Willard van Orman (1976). Grades of discriminability. Journal of Philosophy 73(5), $113-116$.

Saunders, Simon (2003). Physics and Leibniz's principles. In: K. Brading und E. Castellani (Hg.), Symmetries in Physics: Philosophical Reflections. Cambridge: Cambridge University Press.

Saunders, Simon (2006). Are quantum particles objects? Analysis 66, 52-63.

Stachel, John (2002). 'The relations between things' versus 'The things between relations': The deeper meaning of the hole argument. In D. B. Malament (Hg.), Reading Natural Philosophy: Essays in the History and Philosophy of Science and Mathematics, 231-266. La Salle, IL: Open Court.

Teller, Paul (1983). Quantum physics, the identity of indiscernibles and some unanswered questions. Philosophy of Science 50(2), 309-319.

Weyl, Hermann $\left(1928,{ }^{2} 1931\right)$. Gruppentheorie und Quantenmechanik. Leipzig: Hirzel.

Weyl, Hermann (1952). Symmetry. Princeton: Princeton University Press.

Weyl, Hermann (1966). Philosophie der Mathematik und Naturwissenschaft. München: Oldenbourg (3. erweiterte Auflage der erweiterten amerikanischen Ausgabe bei Princeton University Press 1949; 1. Auflage 1928). 